\title{
High performance motion detection: some trends toward new embedded architectures for vision systems
}

\author{
Lionel Lacassagne $\cdot$ Antoine Manzanera $\cdot$ \\ Julien Denoulet · Alain Mérigot
}

Received: 30 June 2007/ Accepted: 2 September 2008/Published online: 14 October 2008

(C) The Author(s) 2008. This article is published with open access at Springerlink.com

\begin{abstract}
The goal of this article is to compare some optimised implementations on current high performance platforms in order to highlight architectural trends in the field of embedded architectures and to get an estimation of what should be the components of a next generation vision system. We present some implementations of robust motion detection algorithms on three architectures: a general purpose RISC processor-the PowerPC G4—a parallel artificial retina dedicated to low level image processingPvlsar34-and the Associative Mesh, a specialized architecture based on associative net. To handle the different aspects and constraints of embedded systems, execution time and power consumption of these architectures are compared.
\end{abstract}

L. Lacassagne $(\varangle)$ · A. Mérigot

Institut d'Electronique Fondamentale (IEF/AXIS),

Université Paris Sud, Orsay, France

e-mail: lionel.lacassagne@u-psud.fr;

lionel.lacassagne@ief.u-psud.fr

A. Mérigot

e-mail: alain.merigot@u-psud.fr

\section{A. Manzanera}

Laboratoire d'Electronique et d'Informatique,

Ecole Nationale Supérieure de Techniques avancées (ENSTA),

Paris, France

e-mail: antoine.manzanera@ensta.fr

J. Denoulet

Laboratoire des Instruments et Systèmes d'Ile de France

(LISIF/SYEL), Université Pierre et Marie Curie, Paris, France

e-mail: julien.denoulet@upmc.fr
Keywords Embedded system · Vision-SoC · RISC . SIMD - SWAR · Parallel architecture .

Programmable artificial retina - Associative nets model . Vision - Image processing - Motion detection .

High performance computing

\section{Introduction}

For more than 30 years, Moore's law had ruled the performance and the development of computers, speed and clock frequency were the races to win. This trend slowly drifted as the processing power of computers reached a seemingly stable value. Other constraints (static current consumption, leakage, less MIPS per gates and less MIPS per Watts) of current technology—-90 and $65 \mathrm{~nm}$-gave researchers an impulse to look for innovative directions to improve efficiency and performance of their architectures. Current challenge is to tackle power consumption to increase systems autonomy. Such technology, like IP core within embedded systems, make the processor frequency adaptable and lead to a finely optimised energy consumption. As image processing and computer vision are very CPU demanding, we focus on the impact of the architecture for a frequently used class of algorithms: the motion detection algorithms.

Three architectural paradigms are compared:

SIMD within a register (SWAR) the impact of the SIMD multimedia extension inside RISC processors, to enhance performance;

Programmable artificial retina one elementary processor per pixel for cellular massively parallel computation and low power consumption;

Associative net impact of reconfigurable graph/net between processors for local and global computations 
and also the impact of asynchronous processors on power consumption.

We focus on the advantages and limitations of these architectures through a set of benchmarks. We also show how to modify the algorithms to take advantage of each architecture's specificities. We provide different performance indexes like speed, energy required and a downclocking frequency to enforce real-time execution. Such indexes provide insight on future trends in computer architecture for embedded systems.

The paper is organized as follow. Section 2 introduces a set of motion detection algorithms: frame difference, Markovian relaxation, Sigma-Delta algorithm and postprocessing morphological operators. Section 3 presents the three architectures: the PowerPC G4, Pvlsar34 (a 200 $\times 200$ Programmable Artificial Retina) and the Associative Mesh (an asynchronous net with SIMD functional units). This section also provides details about how algorithms are optimised in regard to the targeted architectures. Section 4 deals with benchmarking: benchmark of the different algorithms in term of speed and in term of power consumption. To conclude, a synthesis of two extensive benchmarks is provided.

\section{Motion detection algorithms}

As the number of places observed by cameras is constantly increasing, a natural trend is to eliminate the human interaction within the video monitoring systems and to design fully automatic video surveillance devices. Although the relative importance of the low level image processing may vary from one system to the other, the computational weight of the low level operators is generally high, because they involve a great amount of data. Thus, the ability of video surveillance systems to detect a relevant event (intrusion, riot, distress, etc.) is strongly related to the performance of some crucial image processing functions.

Such fundamental processing step is the motion detection, whose purpose is to partition the pixels of every frame of the image sequence into two classes: the background, corresponding to pixels belonging to the static scene (label 0) and the foreground, corresponding to pixels belonging to a moving object (label 1). A motion detection algorithm must discriminate the moving objects from the background as accurately as possible, without being too sensitive to the sizes and velocities of the objects, or to the changing conditions of the static scene. For long autonomy and discretion purposes, the system must not consume too much computational resources (energy and circuit area). The motion detection is usually the most computationally demanding function of a video surveillance system. How the algorithm is actually computed and on which architecture, then become crucial questions.

Three algorithm/architecture pairs will be considered here. In order to compare those very different architectures, we will consider different versions of motion detection algorithms with similar quality but relying on different computational models, some of them being more adapted to one architecture than the other.

The motion detection algorithm can be separated into two parts: time-differentiation and spatiotemporal regularization.

The purpose of the time-differentiation part is to provide, for every pixel $x$ and every time index t: a measure of the temporal variation (the observation) is denoted as $O_{\mathrm{t}}$ and an initial value of the motion binary label is denoted as $\hat{E}_{\mathrm{t}}$. The "frame difference" option is classical and fairly obvious: the temporal derivative is approximated by a difference between consecutive frames, whose absolute value is used as a single motion map (observation) $O_{\mathrm{t}}(x)$ $=\left|I_{\mathrm{t}}(x)-I_{\mathrm{t}-1}(x)\right|$ and the initial value of the motion label $\hat{E}_{\mathrm{t}}$ is obtained by thresholding $O_{\mathrm{t}}$. The "Sigma-Delta" option-detailed in Sect. 2.1-is a recent algorithm [31], based on nonlinear estimation of temporal statistics of every pixel.

The spatiotemporal regularization part aims at exploiting the correlations between neighboring pixels in the motion measures in order to improve the localization of the moving objects. Two main options are considered here (1) morphological filtering, detailed in Sect. 2.2 and (2) Markovian relaxation, detailed in Sect. 2.3.

So, the "Sigma-Delta" can be seen as a pre-processing step for the Markovian regularization or as the main algorithm when followed by a morphological postprocessing.

\subsection{Sigma-Delta estimation}

The principle of the $\Sigma \Delta$ algorithm is to estimate two parameters $M_{\mathrm{t}}$ and $V_{\mathrm{t}}$ of the temporal signal $I_{\mathrm{t}}$ within every pixel using $\Sigma \Delta$ modulations. It is composed of four steps: (1) update the current background image $M_{\mathrm{t}}$ with a $\Sigma \Delta$ filter, (2) compute the frame difference between $M_{\mathrm{t}}$ and $I_{\mathrm{t}}$, (3) update the time-variance image $V_{\mathrm{t}}$ from the difference $O_{\mathrm{t}}$ using a $\Sigma \Delta$ filter and (4) estimate the initial motion label $\hat{E}_{t}$ by comparing the current difference $O_{\mathrm{t}}$ and time-variance $V_{\mathrm{t}}$. 


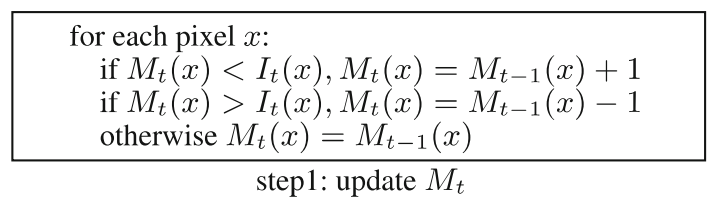

for each pixel $x$ :
$\quad O_{t}(x)=\left|M_{t}(x)-I_{t}(x)\right|$
step2: compute $O_{t}$

for each pixel $x$ such that $O_{t}(x) \neq 0$ :
if $V_{t}(x)<N \times O_{t}(x), V_{t}(x)=V_{t-1}(x)+1$
if $V_{t}(x)>N \times O_{t}(x), V_{t}(x)=V_{t-1}(x)-1$
otherwise $V_{t}(x)=V_{t-1}(x)$
step3: update $V_{t}$

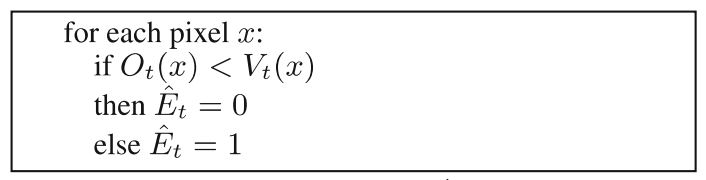

step4: estimate $\hat{E}_{t}$

Apparently, the only parameter is the amplification factor $N$ of the difference (typical values of $N$ are in 2-4). The dimension of $N$ is the number of standard deviation used in the initialization of the motion label. In fact, the updating frequency, which has the dimension of number of gray level per second, can also be adapted. This is a way of customizing the $\Sigma \Delta$ estimation to different kinds of motion and image noise [32].

\subsection{Morphological filtering}

\subsubsection{Alternate sequential filters (ASF)}

The first option of morphological filtering is to perform a sequence of dilatations and erosions using a set of structuring elements of increasing size, such as a sequence of discrete balls $\left(B_{n}\right)_{n}, B_{n}=\left\{z \in \mathbb{Z}^{2} ; d(z, O) \leq n\right\}$, with $O$ the origin of the discrete plane $\mathbb{Z}^{2}$ and $d$ a discrete distance of $\mathbb{Z}^{2}$. Table 1 shows the definitions of such operators.

$\wedge$ and $\vee$, respectively, represent the logical AND and OR. By convention, $\Xi_{0}$ and $\Theta_{0}$ both correspond to the identity function. In this option, the spatiotemporal regularization is performed by applying an alternated sequential filter of

Table 1 Morphological operators

\begin{tabular}{ll}
\hline$\varepsilon_{B}(I)(x)=\wedge_{b \in B} I(x-b)$ & $\gamma_{B}=\delta_{B} \circ \varepsilon_{B}$ \\
$\delta_{B}(I)(x)=\vee_{b \in B} I(x+b)$ & $\varphi_{B}=\varepsilon_{B} \circ \delta_{B}$
\end{tabular}

(a)

(b)

$\begin{array}{ll}\xi_{B}=\varphi_{B} \circ \gamma_{B} & \Xi_{n}=\xi_{B_{n}} \circ \Xi_{n-1} \\ \theta_{B}=\gamma_{B} \circ \varphi_{B} & \Theta_{n}=\theta_{B_{n}} \circ \Theta_{n-1}\end{array}$

(c)

(d)

$a$ Erosion and dilatation, $b$ opening and closing, $c$ alternate filters and $d$ alternate sequential filters certain size to the output of the temporal detection. Typically, $E_{t}=\Xi_{2}\left(\hat{E}_{\mathrm{t}}\right)$.

\subsubsection{Density operators}

In a similar fashion, density operators are defined using a structuring element $B$, except that the binary response is based on counting-thresholding instead of AND-OR combinations :

$\mathcal{D}_{B}(I)(x)=1 \Longleftrightarrow|\{b ; I(x-b)=1\}| \geq \theta$

where $|S|$ represents the cardinality of set $S$ and $\theta$ a threshold representing a required density of $1 \mathrm{~s}$. In this case, the final label is computed using a density operator with a ball of radius $n: E_{t}=\mathcal{D}_{B_{n}}\left(\hat{E}_{\mathrm{t}}\right)$. Typically $n$ equals 1,2 or 3 and usually $\theta=\left\lceil\left|B_{n}\right| / 2\right\rceil$ (majority voting).

\subsubsection{Geodesic reconstruction}

Defined from a binary reference image $R$, the geodesic reconstruction $\operatorname{Rec}^{R}(I)$ of image $I$ within reference $R$ is the relaxation of the geodesic dilatation of $I$ within $R: \delta_{B}^{R}(I)=$ $\delta_{B}(I) \wedge R$. Assuming that the structuring element $B-$ basically a discrete ball of radius 1 -is defining the topology, $\operatorname{Rec}^{R}(I)$ corresponds to the connected components of $R$ having a non-empty intersection with $I$.

In this option, the final label $E_{\mathrm{t}}$ is computed as follows: small connected components elimination using an opening by reconstruction with a ball of radius $n: \tilde{E}_{t}=$ $\operatorname{Rec}^{\hat{E}_{\mathrm{t}}}\left(\gamma_{B_{n}}\left(\hat{E}_{\mathrm{t}}\right)\right)$, then temporal confirmation by computing another reconstruction: $E_{t}=\operatorname{Rec}^{\tilde{E}_{t}}\left(\tilde{E}_{t-1}\right)$.

The final motion label $E_{\mathrm{t}}$ then corresponds to the objects (connected components) bigger than $B_{n}$ that appear on two consecutive frames.

\subsection{Markovian relaxation}

Markov random field based algorithms (MRF) have asserted themselves in a lot of image processing areas for regularizing ill-posed problems. Albeit robust, their wellknown drawback is their CPU consumption due to a large amount of computations, which led researchers to look for solution to speedup its execution time, using parallel machines or dedicated architectures [1, 2, 9, 21, 30].

We follow the MRF model introduced for motion detection purposes proposed by the LIS-Grenoble laboratory [7] and derived from the IRISA model [4, 28]. This model is based on the estimation of a binary (background/foreground) motion field $e$ given an observation field $o$, by maximizing a Bayesian maximum a posteriori criterion, i.e. given a realization of the observation field $o=y$, finding the realization $x$ of the motion label field $e$ that maximizes the conditional probability $P(e=x / o=y)$. Assuming that $e$ is an MRF linked 
to $o$ with a probabilistic relation, this corresponds to finding the motion field $e$ that minimizes the global energy function defined over the set of pixels $\mathbb{S}$ as follows:

$U=\sum_{s \in \mathbb{S}}\left[U_{m}(e(s))+U_{a}(e(s), o(s))\right]$

with $U_{m}(e(s))=\sum_{r \in \mathcal{V}(s)} V_{e}(e(s), e(r))$,

and $U_{a}(e(s), o(s))=\frac{1}{2 \sigma^{2}}[o(s)-\Psi(e(s))]^{2}$.

$U_{m}(e(s))$ is called model energy and is designed to provide spatiotemporal regularity in the motion field. It is based on the Markovian modeling of $e$ as a Gibbs field, where $\mathcal{V}$ is the set of neighbors of the pixel $s$ and the potential functions $V_{e}(e(s), e(r))$ :

$V\left(e_{s}, e_{r}\right)= \begin{cases}-\beta_{s r} & \text { if } e_{s}=e_{r} \\ +\beta_{s r} & \text { if } e_{s} \neq e_{r}\end{cases}$

The $\beta_{s r}$ are positive constants whose values depend on the nature of the neighborhood. We use a uniform 10connected spatiotemporal topology (see Fig. 1), with 3 different values $\beta_{s}=20$ for the 8 spatial neighbors, $\beta_{p}=10$ for the past neighbor and $\beta_{f}=30$ for the future neighbor. Experimental tests demonstrate that these parameters do not have to be tuned according to the image sequence.

$U_{a}(e(s), o(s))$ is called fitness energy and is designed to ensure a certain level of attachment to the input data, i.e. the observation $o$. This term comes from the conditional probability of the observation field $o$, with respect to the motion field $e$, assuming that $o(s)=\Psi(e(s))+n\left(0, \sigma^{2}\right)$, with $n\left(0, \sigma^{2}\right)$ a centered Gaussian noise of variance $\sigma^{2}$, $\Psi(e(s))=0$ if $e(s)$ has the background value and $\Psi(e(s))$ $=\alpha$ if $e(s)$ has the foreground value. The $\alpha$ parameter can be set to usual value 20 , or updated on the fly, as the average value of the moving observations; $\sigma^{2}$, the variance of the moving observation, is computed for every frame.

The minimization of the global energy $U$ is realized by the deterministic relaxation called iterated conditional mode (ICM): all the pixels are sequentially updated and each pixel $s$ is given the label $e(s)$ corresponding to the smallest local energy $U_{m}(e(s))+U_{a}(e(s), o(s))$. Usually, instead of a true relaxation, a limited number of scans is performed (typically 4). The advantage is that the

Fig. 1 Spatiotemporal topology

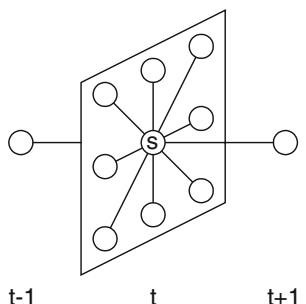

computation time becomes independent of the data, in particular of the initial value of the motion field $e$.

But the drawback is that the quality of the final labeling is very dependent on that initial value, which must be close enough to the final solution. In our algorithm, we use the output of the $\Sigma \Delta$ temporal differentiation $\hat{E}_{t}$, which as proved a good choice of initial guess [31]. The observation field $o$ corresponds to the difference map $O_{\text {t }}$.

\section{Architectures and their optimizations}

In order to perform a fair comparison of these architectures, the algorithm must be optimised for each one. This section describes how the different algorithms are implemented on the three architectures, the impact of the architecture on the algorithm and how the algorithms' structure and the architectures themselves should be modified to obtain optimised implementation.

\subsection{PowerPC}

The powerPC used is a PPC 7447 running at $1 \mathrm{GHz}$. It has a $32 \mathrm{~KB} \mathrm{L1}$ cache, a $512 \mathrm{~KB}$ L2 cache and its power consumption is $10 \mathrm{~W}$. Its specifications are detailed in Tables 5 and 6. From a functional point of view (Fig. 2), it has one Load/Store Unit, one ALU, one FPU and a superscalar SWAR unit: Altivec. Altivec is a multimedia instruction set extension which has been designed to efficiently accelerate image and signal processing [15] applications. Altivec is composed of four 128-bit SWAR units (following the Freescale vocabulary):

- Vector Permute Unit, which handles the instructions to rearrange data within SWAR registers (permutation, selection),

- Vector Simple Integer Unit, which handles all the fast and simple integer instructions,

- Vector Complex Integer Unit, which handles the slower and complex instruction like multiply, multiply-add,

- Vector Floating Point Unit, that handles all the SWAR floating-point instructions.

Main advantages of Altivec are:

- Each of the four vector units are pipelined,

- Two instructions from the four units can be issued per cycle without constraint on which unit is used.

To optimize a given code for a SWAR RISC processor, we have to address the following points:

- bandwidth problem by optimizing loads and data reuse, avoiding data reload and optimizing cache locality [36], 
Fig. 2 PowerPC G4 pipeline

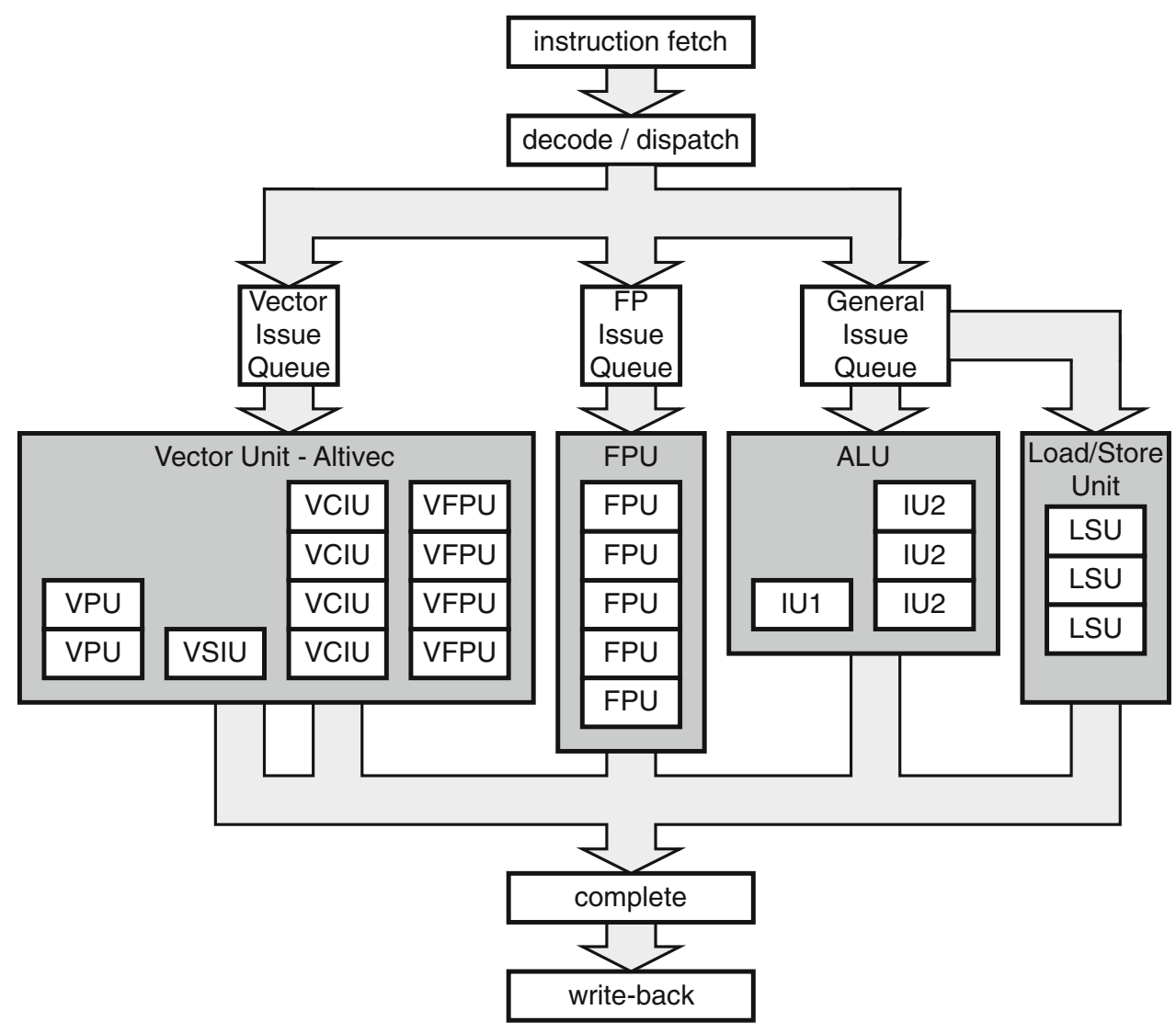

- pipeline stalls due to un-predictable test instructions by trying to remove tests from MRF energy computation,

- pipeline throughput with loop transformations.

In this section, we focus on SWAR optimization (also known as SIMDization) and algorithm transformations. Details about loop transformation techniques and above optimizations are given in [14].

To speedup the computation of the model energy $U_{m}$, we have to transform the equation of the potential function $V$, that comes from the Ising model (the spin associated with a particle). Usually spin-up and spin-down are coded with +1 or -1 . In our case, rather than labeling the state of a site $-1,+1$ for background or motion pixel, we use the binary code 0,1 . Let $p_{1}, s_{1}$ and $f_{1}$ the number of sites, connected to $e_{s}$, with a value 1 , in the past, present and future images $\left(p_{1} \in\{0,1\}, s_{1} \in\{0, \ldots, 8\}, f_{1} \in\{0,1\}\right)$. Then the energy model can be computed without any test or comparison:

$u_{m 1}=\left(8-2 s_{1}\right) \beta_{s}+\left(1-2 p_{1}\right) \beta_{p}+\left(1-2 f_{1}\right) \beta_{f}$,

$u_{m 0}=-u_{m 1}$

where $u_{m 1}$ is the energy associated to a central site at 1 . The fitness energy can also be computed without taking into account the state of the site: $u_{a 0}=\frac{1}{2 \sigma^{2}}[o(s)]^{2}, \quad u_{a 1}=\frac{1}{2 \sigma^{2}}[o(s)-\alpha]^{2}$

If $u_{m 1}+u_{a 1}<u_{m 0}+u_{a 0}$, the state is set to 1 otherwise it is set to 0 . The change is performed whatever the previous state was. The same approach is used to remove tests from the $\Sigma \Delta$ algorithm which is actually very hard to optimize since only a few additions and comparisons are done compared to the amount of memory accesses, as described in [14]. Note that this test can be optimised by rewriting $2 u_{m 1}<u_{a 0}-u_{a 1}$ :

$u_{m 1}<\delta u_{a}, \quad \delta u_{a}=\frac{u_{a 0}-u_{a 1}}{2}=\frac{\alpha(2 o-\alpha)}{4 \sigma^{2}}$

\subsubsection{Density and opening}

We have implemented three kernel size for these operators: $3 \times 3$, for regular use, $5 \times 5$ and $7 \times 7$ to estimate the adequacy of the considered architectures to those well known kernel operators. The cardinal computation of the ball of diameter $k$, i.e. the summation of pixel value over the $k \times k$ kernel (Fig. 3) requires $k^{2}$ LOAD, 1 STORE and $k^{2}-1$ mathematical operations (typically ADD but could be AND or OR Boolean operator for erosion and dilatation). Taking into account that $k \times k$ kernels overlap from one 


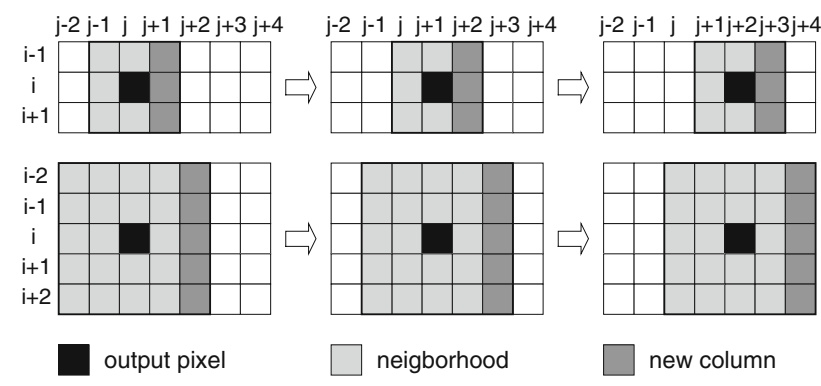

Fig. 3 Operators with neighborhood: overlapping for $3 \times 3$ and 5 $\times 5$ kernels

iteration to another one, such summation can be optimised by splitting this summation into $k$ columns summation. The cardinal is then the sum of these $k$ columns. For the next iteration, only one new column should be computed and added to the previous one. The new complexity is $k$ LOAD, 1 STORE and only $2(k-1)$ ADD (see Table 2).

For SWAR computation, the same optimizations can be applied except that 16 pixels are computed in parallel instead of only one SWAR results are given in Table 2 (for 16 pixels). SWAR implementation requires the construction of unaligned vector registers to compute the partial sums. This is quickly done thanks to the dedicated Altivec instruction vec_sld (Fig. 4). For example, given three

Table 2 Instructions per point, scalar and SWAR version

\begin{tabular}{lll}
\hline Instruction & Without split & With split \\
\hline Scalar LOAD & $k^{2}$ & $k$ \\
Scalar STORE & 1 & 1 \\
Scalar mathematical $O p$ & $k^{2}-1$ & $2(k-1)$ \\
SWAR LOAD & $3 k$ & $k$ \\
SWAR STORE & 1 & 1 \\
SWAR mathematical $O p$ & $k^{2}-1$ & $2(k-1)$ \\
\hline
\end{tabular}

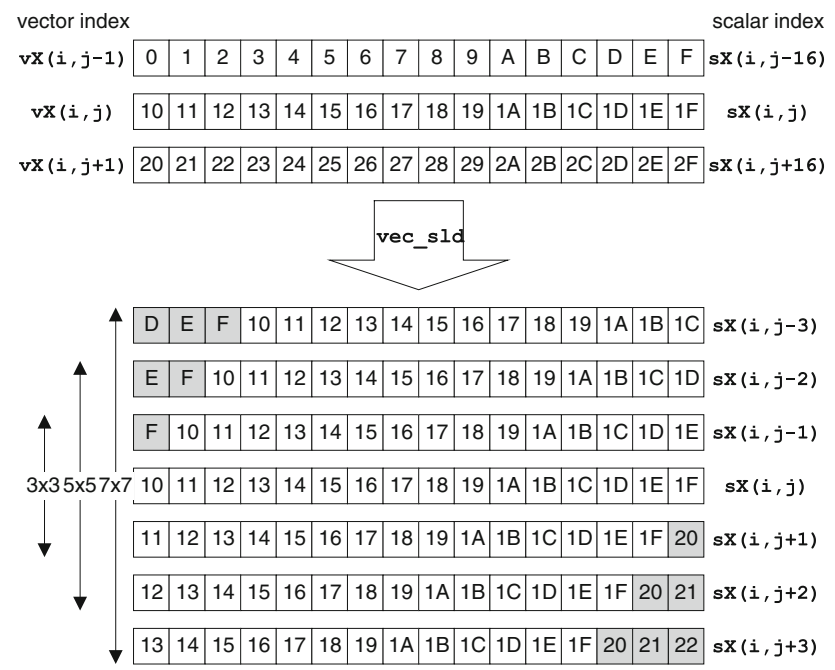

Fig. 4 SWAR convolution reuse aligned vector registers (Fig. 4) $v X(i, j-1), \quad v X(i, j)$, $v X(i, j+1)$, the $k=7$ unaligned vector registers $s X$ are constructed and used to compute the sum of line $i$, (same computations are performed for lines $i-3, i-2, \ldots, i+3)$. The interesting fact is that, up to a value of $n=16$, pixels $x(i, j-n)$ and $x(i, j+n)$ are in the left and right vector registers of $x(i, j)$ (Fig. 4). So, for kernels size up to $k \times$ $k=33 \times 33$, only $3 \times k$ SWAR LOAD are required.

\subsection{Programmable artificial retina}

The purpose of the programmable artificial retina (PAR) project is to develop versatile, real-time, compact and lowpower vision systems. In the vision machines today, most of the resource consumption is due to the transfer of huge amounts of data throughout the different parts of the system. The data flow thus becomes the main source of cost in time, circuit area and/or energy. In PAR-based vision systems, the data transfers are limited to the minimum, by processing the information where it is acquired, i.e. within every pixel of the sensor and by performing an information reduction in the focal plane in order to extract only a few descriptors representing a very small data flow that can be processed by a low-power external processor.

The PAR concept originates from the neighborhood combinatorial processing (NCP) retinas [42] which were SIMD Boolean machines. The near sensor image processing (NSIP) concept [20] then allowed to process gray level images. Now, the deep sub-micron level of CMOS technology allows to put more and more powerful processing circuitry aside the photo receptors while preserving good acquisition performance and resolution [26, 40]. The circuit used in our work was designed by Bernard at ENSTA and fabricated using $0.35 \mu \mathrm{m}$ technology: Pvlsar34 is a $200 \times 200$ retina, with an elementary digital processor and 48 bits of memory within every pixel. The architecture of Pvlsar34 is presented in Sect. 3.2.1, and the retinal algorithms in Sect. 3.2.2 Now, whereas this architecture has proved well adapted to low and medium level image processing [32, 35], the interest of asynchronism has been identified to enhance the processing power of the PARs by providing them with a higher (i.e. regional) level of computation $[18,23,24]$. This is discussed in Sect. 3.2.3.

\subsubsection{Retina and cortex architecture}

The detection algorithm presented in this paper was actually implemented on the architecture presented in Fig. 5 . The PAR Pvlsar34 is a CMOS sensor and a parallel machine at the same time. It is a grid of $200 \times 200$ pixels/ processors connected by a regular 4-neighbors rectangular mesh. The processors execute synchronously, on their local data, a sequence of instructions sent by the controller, 


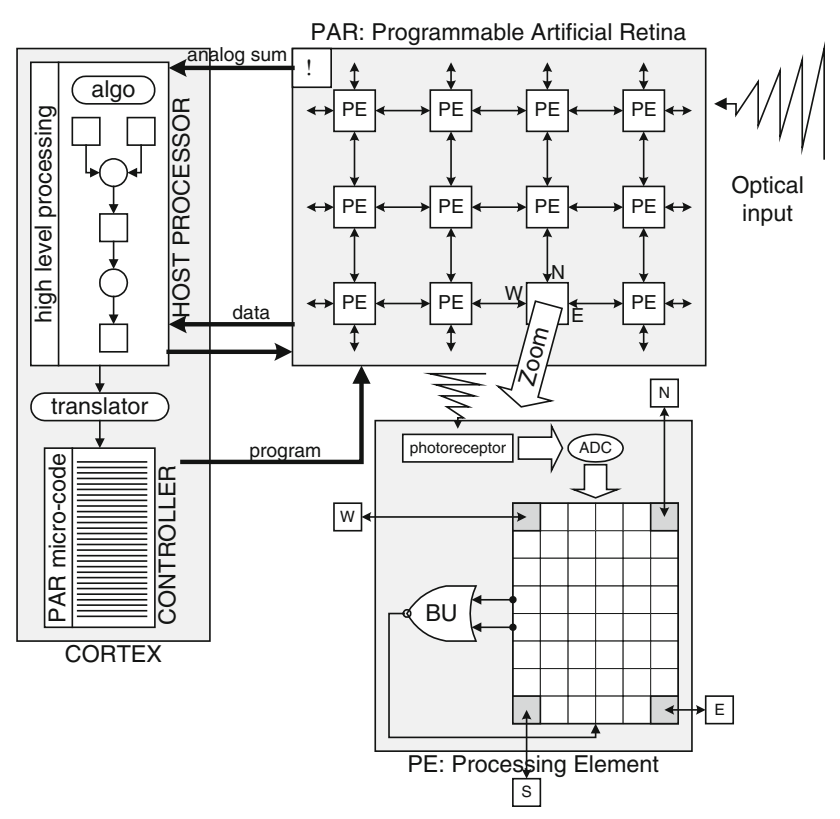

Fig. 5 Architecture of the system composed of the PAR and cortex, with focus on one elementary processor

which is the NIOS processor IP core of the Excalibur FPGA chip. The host processor or cortex is the ARM processor hardware core of the Excalibur. It can exchange data with the PAR, modify the program sent by the NIOS to the PAR and is in charge of the higher levels of computation (i.e. non-image processing) of the vision task.

Every pixel/processor of the PAR is composed of:

- one photo-receptor;

- one analog to digital converter;

- 48 bits of digital memory;

- one Boolean unit (BU), which can read some bits of the digital memory, compute a Boolean operation and write its output on one bit of the digital memory.

The actual instruction set of Pvlsar34 is composed of only five instructions. If $\mathrm{reg}_{1}$ and $\mathrm{reg}_{2}$ are two binary registers of the digital memory:

one: The BU takes the logical value 1.

rd(reg $)_{1}$ : The BU takes the logical value of register reg ${ }_{1}$. $\operatorname{ror}\left(\mathrm{reg}_{1}, \mathrm{reg}_{2}\right)$ : The $\mathrm{BU}$ takes the logical value of the binary $\mathrm{OR}$ between the two binary registers reg $\mathrm{g}_{1}$ and $\mathrm{reg}_{2}$. wd $\left(\mathrm{reg}_{1}\right)$ : The $\mathrm{BU}$ writes down its logical value on register reg $_{1}$.

$\mathbf{w c}\left(\right.$ reg $\left._{1}\right)$ : The BU writes down the complementary of its logical value on register reg $_{1}$.

Boolean algebra shows that this instruction set is sufficient to compute any Boolean function. Now, for readability purposes, we shall use in the presentation of the primitives a generic Boolean instruction set, made of the instructions of the form $y=O P\left(x_{1}, x_{2}\right)$, where $x_{1}, x_{2}, y$ are 3 bits (not necessarily distinct) of the digital memory and $O P$ is any binary Boolean function (e.g. AND, XOR, ADD NOT, etc). Note that every instruction is computed in a massively parallel SIMD mode, the operators are then performed simultaneously on all pixels.

Every pixel of the PAR shares 1 bit of its memory with each one of its four closest neighbors, allowing spatial interactions and image translations. Regarding data extraction, there are two ways to output information from the PAR:

- by translating the image and reading the output on the edge of the grid, to get the exact content of one or more bit planes of the digital memory.

- by using the Analog Global Summer, which provides in constant time an approximate measure of the number of $1 \mathrm{~s}$ in any bit plane of the digital memory.

Although simple, this last feature is important as it provides efficiently global measures that are very useful to get spatial statistics or to detect the convergence of relaxation algorithms.

\subsubsection{Cellular synchronous algorithms}

From the architecture presented above, it turns out that the retinal algorithmics at the present time is essentially a cellular SIMD parallelism. A retinal program is a sequence of binary Boolean instructions. All the pixels/processors perform the same instruction at the same time on their own data, part of which can be taken from one of their closest neighbors. The extreme level of granularity and the small amount of digital memory are the main characteristics of the retinal algorithmic. The algorithm designer is imposed a constant effort of logic minimization, in order to find the Boolean expression of its algorithm that minimizes the number of elementary instructions (related to the computation time) while fitting in the available memory (just like a hardware designer will make a circuit trying to minimize the critical paths and using the minimal amount of logical gates).

Naturally, the memory limitations also affect the data representation that can be used by the algorithm. In the case of motion processing which concerns this paper, this means that the memory used to represent the past history of every pixel must be rigorously controlled. Typically, we shall not keep histograms nor a large set of past values within every pixel, but rather a limited number of temporal statistics, computed recursively.

Despite these constraints, the retinal computation model offers some very attractive features. In particular, the fusion of acquisition and processing functions allows a close adaptation to the lighting conditions and to the scene dynamics. More precisely, the analog to digital conversion (ADC) performed at the output of the photo-receptor is done by a multiple reading which provides $N$ binary images 
(level sets). As the ADC itself is fully programmable, it is possible to perform a constant feedback from the local and global computations to the acquisition, thus providing sophisticated adaptation to lighting conditions.

Once the gray levels of the image are coded within every pixel of the PAR, the retinal program applies a sequence of arithmetic and logic operations that are completely written in software, at the bit level. We now present such program in the particular case of the motion detection.

The $\Sigma \Delta$ change detection algorithm relies on very simple primitives: comparison, difference and elementary increment and decrement. Furthermore, it is based on nonlinear computations which does not involve neither truncation nor dynamics increasing. It is thus well adapted to the minimal instruction set and the small memory of the PAR elementary processors. The implementation on $P v l$ sar34 was performed using the four primitives presented in Table 3. To avoid confusing notation, $I_{\mathrm{t}}$ is noted here $X_{\mathrm{t}}$.

Table 3(3) represents the strict comparison primitive between $X_{\mathrm{t}}$ and $M_{\mathrm{t}}$; $e$ and $f$ are the two bits of result, indicating whether $M_{\mathrm{t}}<X_{\mathrm{t}}$ and whether $X_{\mathrm{t}}<M_{\mathrm{t}}$, respectively. These indicators are used in the $\Sigma \Delta$ algorithm, to update the statistics, by decrementing $e$ (Table 3(4)) and incrementing $f$ (Table 3(5)). Table 3(1) shows the computation of the difference $O$ coded on $n$ bits $\left\{o_{0}, \ldots, o_{n-1}\right\}$, between the current mean $M_{\mathrm{t}}$ and the current sample $X_{\mathrm{t}}$. At the end of the computation, $O_{\mathrm{t}}$ is coded in classical twocomplement, with $c$ the sign bit. For the second-order

Table 3 The PAR algorithmic primitives used in the $\Sigma \Delta$ motion detection

$c=1$
for $\mathrm{i}=0$ to $(\mathrm{n}-1)\{$
$\quad a=m_{i} \oplus \overline{x_{i}}$

$o_{i}=a \oplus c ;$
$a=a \wedge c ;$
$\quad \begin{aligned} & c=m_{i} \wedge \overline{x_{i}} \\ & \\ & c=c \vee a\end{aligned}$

(1) Signed difference

$b=c \wedge o_{0}$
for $\mathrm{i}=1$ to $(\mathrm{n}-1)\{$
$\quad o_{i}=o_{i} \oplus b$
$\quad a=c \wedge o_{i}$
$\quad b=b \vee a$
\}$^{3}$

(2) Absolute value

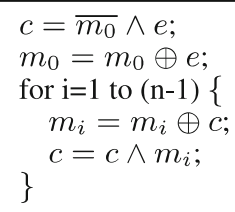

(4) Decrement

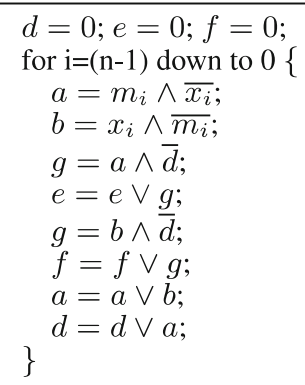

(3) Strict comparison

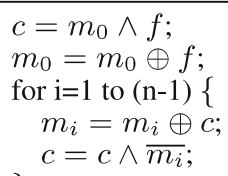

(5) Increment statistics $\left(\Sigma \Delta\right.$ variance $\left.V_{\mathrm{t}}\right)$, it is necessary to compute the absolute value of the difference $O_{\mathrm{t}}$ (Table 3(2)).

The above primitives allow to implement the whole temporal (pixel-wise) part of the algorithm. On Pvlsar34, it was completed by using binary morphology as spatial regularization. An alternate sequential filter was applied on the temporal output $E_{\mathrm{t}}=\Xi_{2}\left(E_{\mathrm{t}}\right)$ (see Sect. 2.2.1). So the only algorithmic primitives that are needed are the logical $\mathrm{OR}$ and the logical ADD between one pixel and its immediate neighbor, in each of the four directions. The filtered output $E_{\mathrm{t}}$ represents the final detection label and it is used as a binary mask to inhibit the update of the $\Sigma \Delta$ mean $M_{\mathrm{t}}$.

The implementation of other spatial operators have been also optimised on the PAR taking care of its constraints (four-connectivity). The 2D filters are split into 1D filters (Fig. 6). There are, at least, two passes: one pass for the vertical operator and one pass for the horizontal operator. After each pass, results are stored into memory. If the operator is not idempotent (like ADD used for the density computation) $(k \times 1)$ and $(1 \times k)$ operators are not split into smaller operators (Fig. 6, top). But if the operator is idempotent (like AND and OR operators used for ASF), each $(k \times 1)$ and $(1 \times k)$ operator is split into a set of $(3 \times 1)$ and $(1 \times 3)$ operators (Fig. 6, bottom), with, at each time, a memory access. This decomposition reduces memory access to directly connected neighbors.

Thus, for $k>3$, non-idempotent $k \times k$ operators are expensive to implement. There are two reasons: the first one is, the great amount of cycles dedicated to gather far pixels to the current PE and the second one is the cost of serial-bit $A L U$ operations. For these reasons the density filter is much more slower than the erosion/dilatation filter $(\times 4.5, \times 5.9$ and $\times 6.3$ slower, respectively). As ASF are based on erosions and dilatations, their implementation remains efficient even if they have a great complexity, making them faster than density operator.

non idempotent operator (ADD)

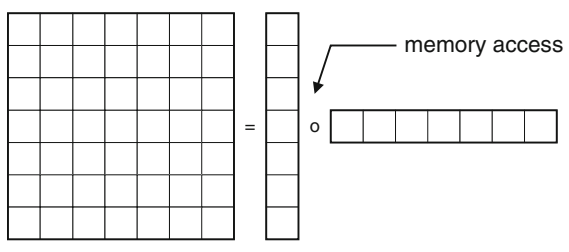

idempotent operator (ADD, OR)

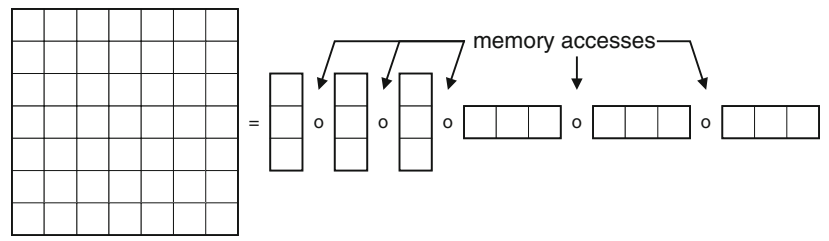

Fig. 6 2D Spatial filters optimization on retina 


\subsubsection{Hybrid algorithms}

Although Pvlsar34 can simply and quickly compute relaxation operators such as skeletons, or morphological connected operators, its efficiency in terms of useful computation is low for such irregular operators because of the expense due to the synchronous sequencing of the whole grid, that will only serve in some specific regions of the image. For that reason, a reduced set of asynchronous operators has recently been proposed by [24] to increase the computing level of the PARs. Thus, programmable connections, spanning tree constructions, OR and SUM asynchronous associations will be integrated in the next generations of PARs.

Such hybrid synchronous/asynchronous architecture will allow us to perform operations over a selected region (connected component) very efficient. This is the case of the geodesic reconstruction, which is useful for the motion detection algorithm (see Sect. 2.2). In the asynchronous model, the corresponding operation is computed like in the Associative Mesh (see Sect. 3.3): the reference set $X$ is used as a binary mask to open/close the connections of the programmable mesh. Then an $\mathrm{OR}$ - association is computed on the marker set $Y$; the output is the result of the reconstruction $\operatorname{Rec}^{X}(Y)$.

\subsection{Associative Mesh}

The Associative Mesh [19] intends to exploit a massive dataparallelism, originating from a model based on network reconfigurability: the Associative Nets Model [33]. To allow efficient hardware optimizations for the large diversity of algorithms in image processing [34], the architecture is built from the observation of data-movements and data-structures encountered in this field. The Associative Mesh relies on a dynamic reconfigurability of its processors network and on an asynchronous electronic used to perform global operations and communication tasks. Reconfigurability and asynchronism offer solutions to adapt architectures to this context [5]. Several studies have shown that most techniques of image processing can be implemented using the Associative Mesh $[3,8,16,17]$ or architecture using some of the implementation techniques of the Mesh and the Associatives Nets concepts [22, 23].

\subsubsection{Associative Nets Model theory}

The Associative Nets Model is characterized by the application of associative operators on a locally reconfigurable, directed interconnection graph called mgraph implemented locally in each processor to enable its dynamic evolution in the course of an algorithm. Mgraphs can represent objects (Fig. 7) coded, processed or

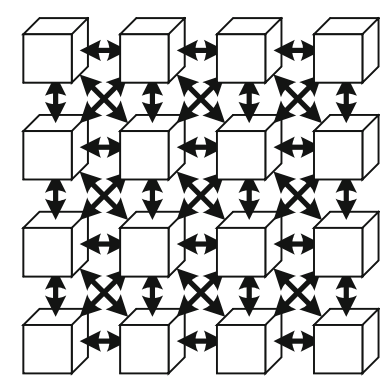

(a)

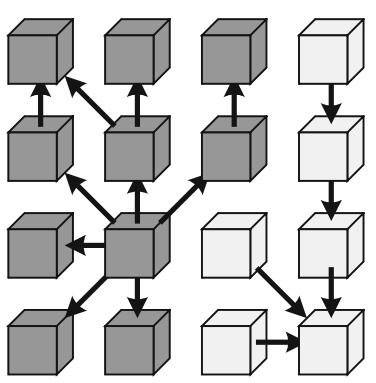

(c)

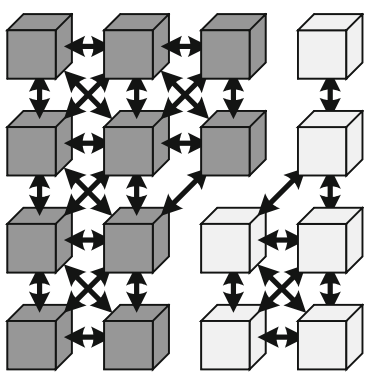

(b)

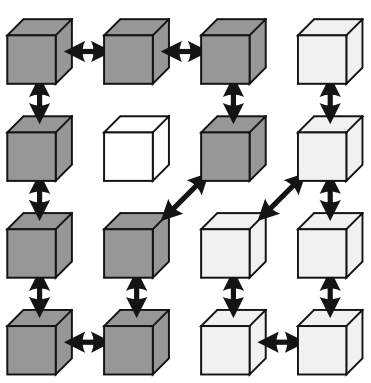

(d)
Fig. 7 Different mgraphs configurations: a full graph; b region graph; c oriented trees and $\mathbf{d}$ edges graph

manipulated in image processing such as connected areas, edges, oriented trees, etc. It allows us to think not only in terms of point-to-point communication between processors but to apprehend information at a higher level.

Operations in the Associative Nets Model combine communication and computation aspects and are called 'associations'. They consist in a global application of an operator-such as logical operators, addition, minimum/ maximum or spanning tree generation-on data spread over a connect set of the considered mgraph. As a basic example, this primitive can be used to asynchronously compute the area of a region by globally summing 1 per pixel on the mgraph connected components. It happens that most complex algorithms can be realized by iterating these primitive operations. Local associations are also allowed and are named Step Associations; the operator in this case is used to combine the local value of a processor with its nearest neighbors on the mgraph. Figure 8 presents an example of a global MAX - association.

\subsubsection{Associative Mesh architecture}

The Associative Mesh is a SIMD hardware transposition of the Associative Nets Model, featuring an 8-connected 2D mesh. Its originality comes from an asynchronous implementation of associations: the interconnection graph can be seen as an asynchronous path where data freely circulate from a processor to another, propagating local results to neighbors until global stability is reached. 


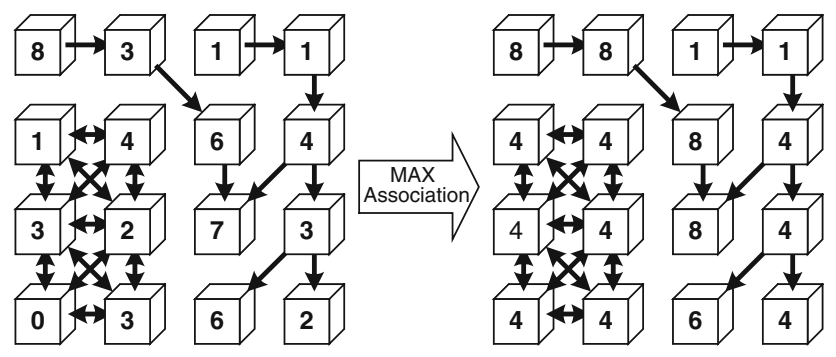

Fig. 8 MAX-association

Reconfigurability directly stems from the concept of mgraphs: each processor includes an 8-bit mgraph register, where each bit emulates the absence or presence of an incoming edge originating from a neighboring processor. The mgraph register is connected to the input of an AND-gate mask, which filters data emitted by the neighbors.

A Mesh processor is built around two distinct parts: an Associative Element (AE) which performs asynchronous associations and a Processing Element (PE) dedicated to internal operations and memory tasks, featuring an all-purpose memory bench, dedicated registers to save the local mgraph value, an independent scan-register for image input/ output and an ALU to perform basic local operations (Fig. 9).

In order to save space, AEs have a 1-bit data-path. A $n$ bit association will then be performed as an iteration of 1bit associations. Operators have been designed to ensure that data cross a minimum of logical layers to optimize the traversal time of each AE. As an example, in a simulation based on a 90-nm technology and a Mesh running at $500 \mathrm{MHz}, 40 \mathrm{AEs}$ can be crossed in one clock cycle during an $\mathrm{OR}$ - association. As a result, the basic global primitives of the model, associations, are performed in a very interesting computation time: simulations using the same technological parameter indicate that for a $512 \times 512$ image, OR - association on 8-bit data is performed in $60 \mathrm{~ns}$ and PLUS - associations in $200 \mathrm{~ns}$ [13]. Such a speed on

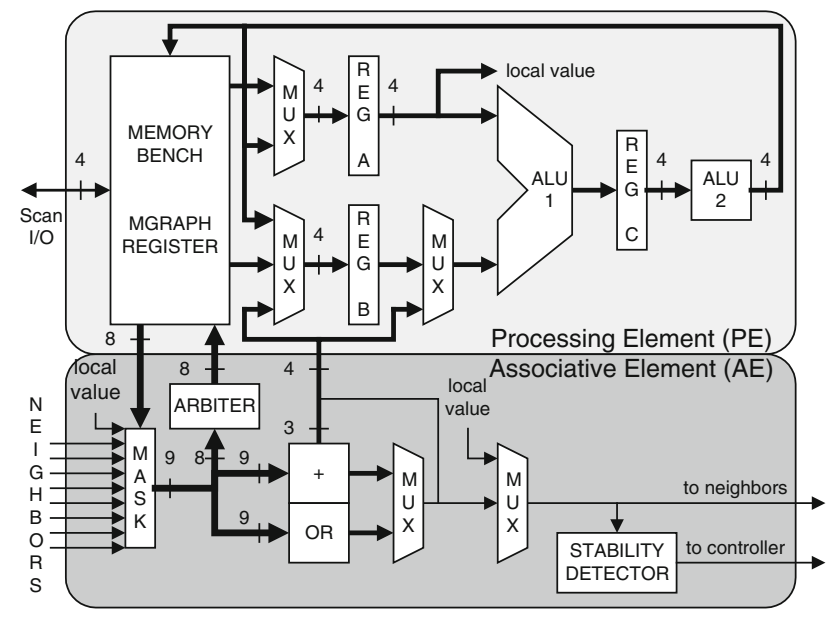

Fig. 9 Processor architecture
Table 4 List of SIMD instructions on the Associative Mesh

Affectation
Equal
Boolean instructions
OR, AND, XOR, NOT, Shift
Comparisons
$=, \neq,>, \geq,<, \leq$
Arithmetical operations
+ , minus $;, \times, /$, modulo

global operations emphasizes the impact of the asynchronous network on the Mesh's performance. Available instructions are listed in Table 4.

\subsubsection{Processor virtualization and SIMD}

With current technologies, the architecture discussed above is not optimised with a SoC approach, meaning a complete image analysis machine inside one chip. We can improve the Mesh integration by changing the PEs granularity: we now assign a group of $N$ pixels to each processing element (now called SIMD PE) and consider that we have $N$ virtual PEs per physical SIMD PE ( $N$ is called degree of virtualization) [12]. To retain the benefits of asynchronism (very fast computation time, easy controllability), the AE structure is preserved in its original configuration. Thus, only the synchronous parts of the design are affected by the virtualization process. Figure 10 presents a virtualized PE dealing with 2 pixels.

This reorganization allows us to envision the architecture as the juxtaposition of an asynchronous communication network and a set of virtualized synchronous units, each managing $N$ pixels. This new structure enables a significant area gain: we have shown that the design area is reduced by $20 \%$ if $N=16,25 \%$ if $N=1,024$. With $N=1,024$, the hardware cost of a $256 \times 256$ Associative Mesh, including 64 SIMD PEs, each managing $32 \times 32$ pixels, is about 165 millions of transistors. However, virtualization induces an increase of computation time due to the serialization of local operations. Still, this increase can be limited by implementing a SIMD unit in each SIMD PE, so we can parallelize, up to a certain point, operations for pixels managed by the same SIMD PE and reduce computation times in significant proportions [13].

\subsection{4 $\Sigma \Delta$ initialization}

The $\Sigma \Delta$ initialization is entirely performed by the SIMD PEs. Parallel conditional statements like WHERE or ELSEWHERE implement the IF - THEN-ELSE instructions by performing a sequence of operations in each PE, according to the result of a local logic comparison. 


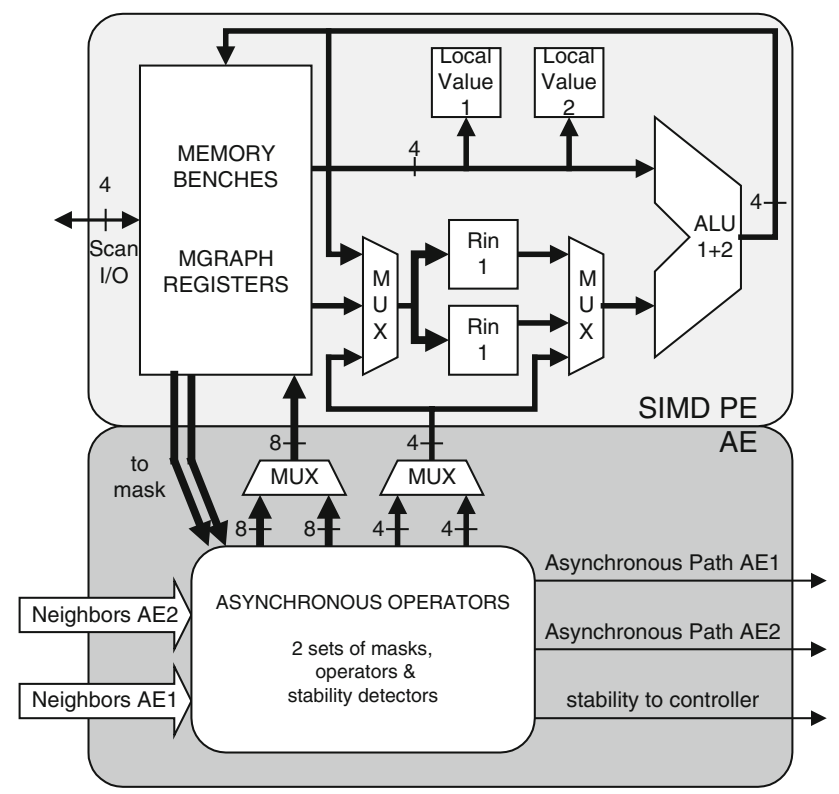

Fig. 10 Processor architecture with virtualization

\subsubsection{Markov}

The update strategy used is image recursive for full-parallel updates. The energies computation are held by the SIMD PEs while the AEs are used to compute $p$, the sum of spatial 8-connected sites, using a local PLUS - association. Conditional statements are used to collect sites label from $E_{\mathrm{t}-1}$ and $E_{\mathrm{t}+1}$, compute $V_{p}$ and $V_{f}$ and also to set the final label to the site, depending on the total energy $u$. Note that the graph mask is configured by the set of the eight masks (the four principal directions: North, South, West, East and the four secondary directions North-West, North-East, South-West and South-East).

// Difference of Adequacy energy computation

$\Delta U_{a}=\left(\alpha \times(2 \times o \times \alpha) / 4 \times \sigma^{2}\right.$;

// Energy due to potential $V_{p}$

$\operatorname{WHERE}\left(E_{t-1}==1\right) \quad U_{p}=-\beta_{p}$;

ELSEWHERE $U_{p}=\beta_{p}$; ENDWHERE;

// Energy due to potential $V_{f}$

$\operatorname{WHERE}\left(E_{t+1}==1\right) \quad U_{f}=-\beta_{f}$;

ELSEWHERE $U_{f}=\beta_{f}$; ENDWHERE;

// Energy due to potential $V_{s}$

// Graph configuration: fully 8-connected graph

$\mathrm{Graph}=\mathrm{mNW}+\mathrm{mW}+\mathrm{mSW}+\mathrm{mS}+\mathrm{mSE}+\mathrm{mE}+\mathrm{mNE}+\mathrm{mN}$;

$\mathrm{s}=$ PLUS-ASSOCIATION $($ Graph $\left.), E_{t}\right)$;

$U_{s}=(8-2 s) \times \beta_{s}$;

// Model energy computation

$U_{m 1}=U_{s}+U_{p}+U_{f}$

// Pixel labeling

WHERE $\left(U_{m 1}<\Delta U_{a}\right) \hat{E}_{t}=1$;

ELSEWHERE $\hat{E}_{t}=0$; ENDWHERE;

Associative Mesh ICM version

\subsubsection{Binary geodesic reconstruction}

Reconstruction takes an efficient use of the Mesh's AE units: the geodesic mask is represented as a graph, where each object is a unique connected component. Pixels of the mask (set to 1) are linked together with the LINK - WITH ONES mgraph creation primitive. The markers are then dilated up to the mask's limits by performing a global $\mathrm{OR}-$ association on the graph. The worst case is met when a unique object—shaped as a spiral-with a marker on one of its extremities fills the $200 \times 200$ image. Simulations based on a $90-\mathrm{nm}$ technology reveal that for this extremely rare configuration, this operation on a Mesh running at $500 \mathrm{MHz}$ will take 500 cycles. However, since data are 1bit wide, it will only take a handful of cycles in most cases for the association to complete, thus providing a very interesting computation time.

// Creation of a graph representing the geodesic mask
// from the ImageMask binary image
LINK-WITH-ONES (GraphMask, ImageMask);
// Markers dilatation
Result=OR-ASSOCIATION (GraphMask, ImageMarker);

Binary geodesic reconstruction on Associative Mesh

\subsubsection{Morphological opening}

A dilatation on binary data, with a $3 \times 3$ structuring object, is simply achieved by a local $\mathrm{OR}$ - association. Operating with a $5 \times 5$ or $7 \times 7$ structuring object only requires an iteration of local associations. Erosion is computed in a similar way, this time with an AND - association. Therefore, a morphological opening will be implemented on the Mesh by computing 1,2 or 3 local AND - association, followed by 1,2 or $3 \mathrm{OR}$ - association, depending on the size of the object.

\subsubsection{Density operator}

On a $3 \times 3$ window, each pixel's eight neighbors are summed in parallel by a local PLUS - association. The final threshold is performed in the SIMD PEs. To operate on larger windows, we must ensure that each pixel will be counted once and once only. Addition is not idempotent, so it is impossible to simply iterate local associations as we did with the morphological opening. In consequence, we have to divide a $5 \times 5$ or $7 \times 7$ window into $3 \times 3$ or smaller sub-windows (Fig. 11). A sub-total is then computed in each sub-window, using a local PLUS - association. Finally, each sub-total is sequentially propagated to the central node to be added in the final sum. A last threshold is then performed in the SIMD PEs. As Boolean associations 


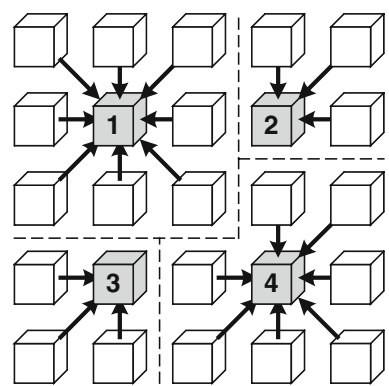

(a)

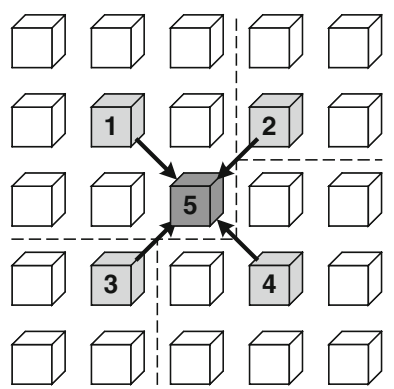

(b)
Fig. 11 a Sub-window split for a $5 \times 5$ density operation; b subtotals propagation to the central node

like $\mathrm{OR}$ - association and AND - association are idempotent, they require less graph configurations and associative operations. For the opening, respectively, 2 and 3 for $5 \times 5$ and $7 \times 7$ associative operators and only 1 graph configuration versus 5 and 17 associative operators and as many as graphs configurations for non-idempotent operation like the addition for the density operator.

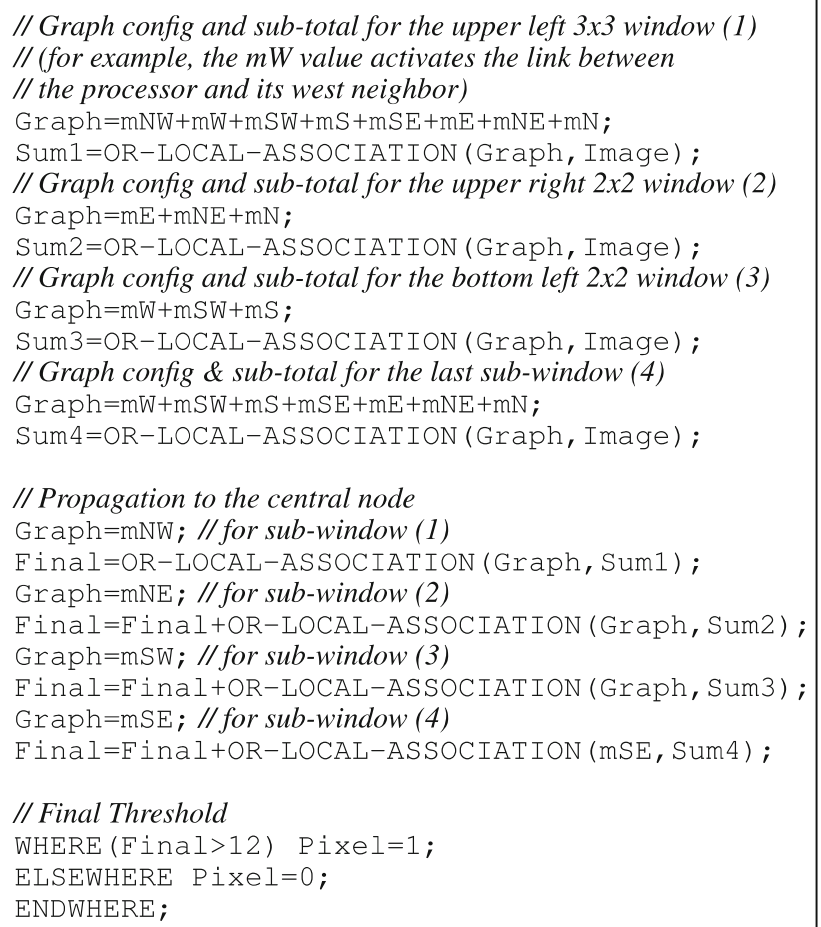

\subsection{Architectures specification summary}

In order to compare the three architectures and to focus on their advantage and drawback, their specifications are summed up into two tables: the architectural specifications
Table 5 Architectures specifications

\begin{tabular}{lllll}
\hline Architectures & Frequency & Internal RAM & Transistors & Watts \\
\hline AM & $500 \mathrm{MHz}$ & $2 \mathrm{MB}$ & $160 \mathrm{M}$ & $2 \mathrm{~W}$ \\
Retina & $5 \mathrm{MHz}$ & $225 \mathrm{~KB}$ & $4 \mathrm{M}$ & $100 \mathrm{~mW}$ \\
$\mathrm{G} 4$ & $1 \mathrm{GHz}$ & $32 \mathrm{~KB}+512 \mathrm{~KB}$ & $58 \mathrm{M}$ & $10 \mathrm{~W}$ \\
\hline
\end{tabular}

(RAM, amount of transistors and power consumption) and bandwidth specifications (access to internal data and external data).

Table 5 provides the size of the internal RAM (size of the cache hierarchy on PowerPC G4 and size of the distributed memory on the Mesh and the retina) and an estimation of the power consumption. For the PowerPC $\mathrm{G} 4$, this is an average value, for the Mesh this is an estimation and for the retina, this is the measured value.

One very important point for comparison is the bandwidth of these architectures. As the Mesh and Retina are parallel architecture, we use the concept of aggregate bandwidth originating from high performance computing. The aggregate bandwidth is the sum of the bandwidth of all processors (Table 6). Then we consider the internal bandwidth as the bandwidth between the processor and its closest RAM (L1 cache for the PowerPC G4 and distributed internal RAM for Mesh and Retina) and the external bandwidth as the bandwidth of the external bus, connecting the processor, to the external RAM or to another processor. For the Mesh, this is the capability of the asynchronous network to transfer data from one AE to another AE. For the Retina this is the bandwidth to transfer data from one memory bank associated to one processor to one of its connected processors. The reason is that, for the retina, the bandwidth cannot be computed in the same way than for RISC processor or an associative network, where internal and external buses can be easily identified. Each elementary processor (PE) of the retina has 48 bits of memory and 4 bits are shared with the four neighbors. Internal bus bandwidth capacity is based on the number of cycles for a READ, i.e. 6 cycles. External bus capacity is the number of cycle to perform a copy from one of the four bits ( 6 cycles for the READ) to one of the 44 private bits ( 3 cycles for the WRITE). Internal bus bandwidth is to access private memory, external bus to access shared

Table 6 Bandwidths, per cycle and per second

\begin{tabular}{lll}
\hline Architecture & External bus & Internal bus \\
\hline AM & $64 \mathrm{~B} / \mathrm{c}$ & $1,024 \mathrm{~B} / \mathrm{c}$ \\
AM & $30 \mathrm{~GB} / \mathrm{s}$ & $476 \mathrm{~GB} / \mathrm{s}$ \\
Retina & $555 \mathrm{~B} / \mathrm{c}$ & $833 \mathrm{~B} / \mathrm{c}$ \\
Retina & $2.8 \mathrm{~GB} / \mathrm{s}$ & $4.1 \mathrm{~GB} / \mathrm{s}$ \\
G4 & $1 \mathrm{~B} / \mathrm{c}$ & $16 \mathrm{~B} / \mathrm{c}$ \\
G4 & $1 \mathrm{~GB} / \mathrm{s}$ & $16 \mathrm{~GB} / \mathrm{s}$ \\
\hline
\end{tabular}


memory. Note that for the Mesh, the bandwidths are computed for an architecture of $256 \times 256$ AEs with a virtualization $N$ of 1,024, i.e. 64 SIMD PEs.

We can notice and it is one of the main advantage of specialized architectures, that both Retina and Mesh can transfer much more data per cycle than a generalist RISC processor ( $\times 64$ for internal and external buses). When considering bandwidth per second, the total aggregate bandwidth of the Mesh is close to the latest Cray vector processor performance [10] which has a peak bandwidth of $800 \mathrm{~GB} / \mathrm{s}$. Keeping in mind that most of the image processing algorithms are faced with memory wall problem, it is like if RISC still wait for data when the distributed buses of specialized machine can transfer data in time to feed processors.

\section{Benchmarks}

In order to compare the architectures, both from a qualitative and quantitative point of view, we used the frame rate and the cycle per point (cpp):

cpp $=\frac{t \times F}{n^{2}}$

where $t$ is the execution time, $F$ the processor frequency and $n^{2}$ the number of pixel to process, per processor. The cpp is an architectural metric to estimate the adequacy of an algorithm to an architecture [27]. For each architecture, we provide the cpp and the speedup for every operator $(\Sigma \Delta$, ICM, morphological operator) and also for the whole algorithm as described in the first section. The algorithms have been implemented on a PowerPC G4 and PAR and have been simulated on the Associative Mesh with SystemC. For parallel architectures, the cpp expression is modified, depending on the number of pixels to be processed by a processor:

$\mathrm{cpp}_{\mathrm{PAR}}=t \times F, \quad \mathrm{cpp}_{\text {Mesh }}=\frac{t \times F}{n^{2} / N}$

The cpp values have been calculated for $128 \times 128,256$ $\times 256,512 \times 512$ and $1,024 \times 1,024$ image size to analyze the cache behavior. We only provide the results for 256 $\times 256$ image size to reduce the amount of results. For specialized parallel architecture like PAR or Mesh, the scalability is quite ideal so extensive results will not provide more information. For the PowerPC, more detailed results are provided to focus on the problem of cache misses.

\subsection{Benchmark procedure}

For the PowerPC, we used the following approach. As there is no clock cycle 64-bit counter, on powerPC under
MacOS, we have used a micro-second counter based on the micro kernel MACH. As execution time is very short for small images, the measure is done on $i$ iterations of the loop, to get a duration of $\times 1,000$ the resolution of the timer. As this measure can be polluted by the OS, $r$ runs are performed, and the minimum is selected.

For the PAR, a logic analyzer Agilent 1670 has been used. Acquisition, conversion and computation time are readable on the analyzer. The figures only take into account the computation time.

For the Mesh, algorithms have been implemented and simulated using a Mesh simulator based on a SystemC description of the architecture allowing a cycle-accurate evaluation. In order to achieve this feature, we need to evaluate the duration of an association. Besides technological or architectural issues, this duration depends on the initial value of the data and of the graph type used for the operation. For instance, an OR-based-association computation time is given by the longest distance between two logical 1s, Therefore, estimation can be performed by computing the number of processors walked through by data during the operation. To implement this process on the Mesh simulator, data circulating in the asynchronous network provide two informations, each going through a specific data path: on one hand, the local result of the association as a 1-bit value uses the standard architecture data path and, on the other hand, a counter representing the number of processors walked through so far by this data, which is incremented after going through a processor (Fig. 12). When the association terminates, data in the network with the highest counter value gives the duration of the association.

\subsection{PowerPC G4 results}

Four algorithms have been benchmarked: ICM, $\Sigma \Delta$, density filters for $3 \times 3$ and $7 \times 7$ kernels and also Frame Difference (FD) algorithm. We added FD to get a reference in term of complexity and then in term of cpp, since no algorithm can be simpler than an absolute difference

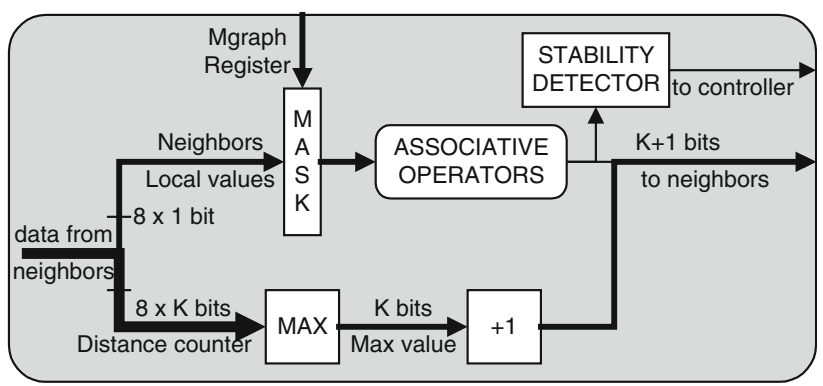

Fig. 12 Asynchronous data path on Associative Mesh simulator 
followed by a threshold to detect motion. For each algorithm, two scalar versions and two "vector" versions were coded:

$s_{0} \quad$ scalar with no optimization, straight-forward coding,

$s_{1} \quad$ scalar with all possible optimizations,

$v_{0} \quad$ SWAR vector version with no optimization,

$v_{1} \quad$ SWAR with optimization like Loop unrolling, Register Rotation, strength reduction and computation factorization.

We provide cpp for classical image size, to point out the problem of cache behavior. For each algorithm, four ratios are also calculated:

$s_{0} /$ the impact of scalar optimization,

$s_{1}$

$v_{0} /$ the impact of SWAR optimization,

$v_{1}$

$s_{1} /$ the impact of SWAR switch for both optimised $v_{1} \quad$ versions,

$s_{0} /$ the total acceleration from a basic/naive code to an $v_{1} \quad$ optimised SWAR code.

We can see that the global speedup $(s 0 / v 1)$ is huge: from $\times 17$ for $\Sigma \Delta$ to $\times 60$ for $7 \times 7$ density filter. We can notice too that the code vectorization is the optimization technique that provides the highest speedup (line $s_{1} / v_{1}$ : from $\times 6.8$ for ICM to $\times 15.6$ for density filter, while the scalar techniques all together provide a speedup $\left(s_{0} / s_{1}\right)$ from $\times 1.2$ for $\Sigma \Delta$ to $\times 6.6$ for ICM. Such value of speedups make the use of optimization and vectorization to assert themselves for real-time computing on generalist purpose SWAR RISC processor.

Note that all the versions $s_{1}, v_{0}, v_{1}$ require some expertise from the developer. If these versions have been compiled with all optimization options of the compiler, without a little help, the compiler cannot achieve a level of performance higher than the $s_{0}$ version.

If we look in detail at the Fig. 13 that represents the cpp's evolution of ICM and $\Sigma \Delta$, for image sizes varying from $128 \times 128$ to $1,024 \times 1,024$, we can focus on two points. First there is a big gap in performance when image size increases and data do not fit in the cache. This phenomenon appears for different image size, depending on the algorithm (about $250 \times 250$ for $\Sigma \Delta$ and $350 \times 350$ for ICM). Then if both cpp are similar in the left part of the figure for small image sizes, the $\Sigma \Delta$ cpp becomes $40 \%$ bigger than ICM cpp. The cpp value is multiplied by $\times 3.8$ between left and right part of the figure. This result is in contradiction with any complexity analysis: $\Sigma \Delta$ is more simple than ICM, but because it requires more images to be present at the same time in the cache and also because there is very few instruction to optimize, there is no possibility to optimize the code. The $\Sigma \Delta$ algorithm is a typical case of memory bounded problem. The performance decrease is

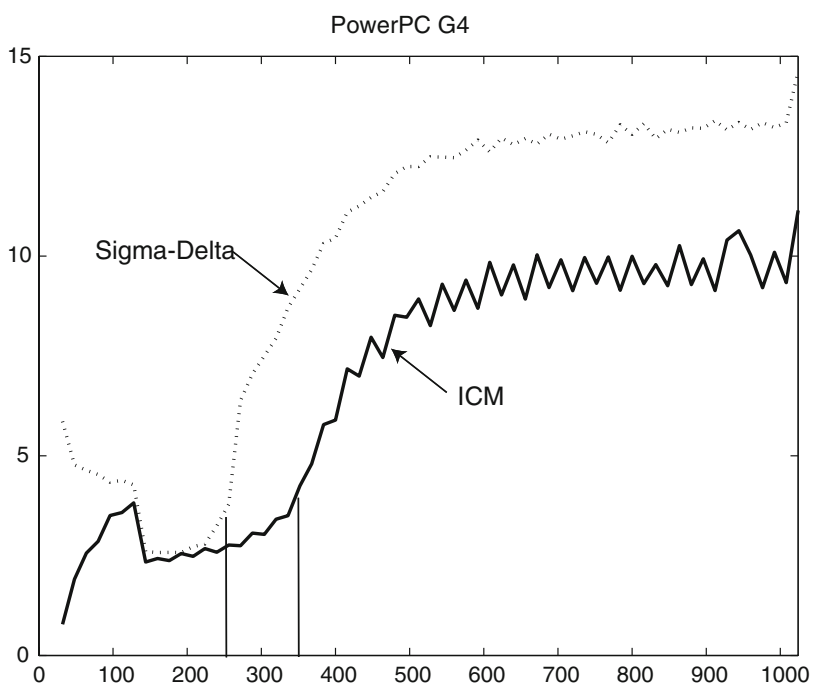

Fig. 13 ICM and $\Sigma \Delta$ cpp on PowerPC G4

more important than for ICM or other algorithms studied here. That raises another problem: SIMDization is efficient only when data fit in the cache, if the global speedup $\left(s_{0} / v_{1}\right)$ is $\times 17.1$ for $256 \times 256$ images size, it is only $\times 5.2$ for $1,024 \times 1,024$ images size.

Finally, if we compare the cpp of the best version $\left(v_{1}\right)$ of ICM or $\Sigma \Delta$ algorithm with the naive scalar version $\left(s_{0}\right)$ of FD or even the optimised scalar version $\left(s_{1}\right)$, we can see that the SIMDization makes complex algorithms like Markov Random Field relaxation, or $\Sigma \Delta$ filtering run faster than FD. From a qualitative point of view, this enforces the use of SWAR on general purpose RISC computer since such SIMD multimedia instructions make robust algorithm run faster than naive algorithm if this one is not optimised.

In the next subsection, we will focus on the implementation of these algorithms on the Retina and on the Mesh to finally compare them from an embedded point of view: frame rate and power consumption.

Table 8 shows, for PowerPC G4, that scalar optimizations are as important as SWAR optimizations: $\times 7$ ! As usual, the most efficient optimization is the highest level optimization: the algorithmic transform by LUT utilization provides a speedup of $\times 3.6$. Caches have also an important impact on performance whether the data fits in the cache $(256 \times 256)$ or not $(512 \times 512$ and more $)$.

\subsection{Retina benchmarks}

The results presented in this section, related to computation time and energy, have been measured on our experimental device composed of the $200 \times 200$ PAR Pvlsar34 connected to an Excalibur board EPXA1, used to control the PAR and to perform higher level computations. The measures have been made using an oscilloscope and a logic 
analyzer except for density operator where figures are estimated, not measured.

Table 9 details the cost in time of the different functions. The first column represents the number of Boolean instructions, the second column the number of clock cycles and the third column the time, in ms. The acquisition corresponds to the time of photo-transduction, during which no operation is performed. This time, measured here in normal conditions of our laboratory, naturally varies according to the lighting conditions. For the following functions (digital conversion, $\Sigma \Delta$ estimation and spatial

Table 7 Implementation on PowerPC G4: cpp and gain

\begin{tabular}{lcrrrr}
\hline Algorithm & FD & \multicolumn{1}{c}{$\Sigma \Delta$} & ICM & Density3 & Density7 \\
\hline \multicolumn{4}{l}{ cpp of scalar } & and vector & versions \\
$s_{0}$ & 28.7 & 50.5 & 112.2 & 27.2 & 114.0 \\
$s_{1}$ & 17.5 & 40.5 & 16.9 & 12.3 & 30.1 \\
$v_{0}$ & 3.4 & 4.5 & 3.4 & 1.9 & 5.7 \\
$v_{1}$ & 1.2 & 3.0 & 2.5 & 1.5 & 2.5 \\
Gain between & scalar and vector versions & & \\
$s_{0} / s_{1}$ & $\times 1.6$ & $\times 1.2$ & $\times 6.6$ & $\times 2.2$ & $\times 3.9$ \\
$v_{0} / v_{1}$ & $\times 2.9$ & $\times 1.5$ & $\times 1.4$ & $\times 1.2$ & $\times 2.7$ \\
$s_{1} / v_{1}$ & $\times 15.0$ & $\times 13.7$ & $\times 6.8$ & $\times 8.2$ & $\times 15.6$ \\
$s_{0} / v_{1}$ & $\times 24.6$ & $\times 17.1$ & $\times 44.9$ & $\times 18.3$ & $\times 60.0$ \\
\hline
\end{tabular}

Table 8 PowerPC G4 optimizations impact for ICM

\begin{tabular}{lll}
\hline Version & $\mathrm{cpp}$ & Gain \\
\hline Basic & 147 & - \\
LUT & 41 & $\times 3.6$ \\
Internal loop unrolling & 32 & $\times 1.3$ \\
External loop unrolling & 20 & $\times 1.6$ \\
SIMD vectorization & 2.6 & $\times 7.7$ \\
\hline
\end{tabular}

Table 9 Computation costs of the different algorithmic functions of $\Sigma \Delta$ detection on Pvlsar34

\begin{tabular}{lrll}
\hline Function & $\# i$ & $\# c$ & $t(\mathrm{~ms})$ \\
\hline Acquisition & 0 & 0 & 15 \\
Digital conversion & 64 & $2.5 \mathrm{k}$ & 2 \\
$\Sigma \Delta$ Estimation & 160 & $6.5 \mathrm{k}$ & 1.3 \\
Spatial binary morphology & & & \\
$3 \times 3$ ASF & 25 & $1 \mathrm{k}$ & 0.2 \\
$5 \times 5$ ASF & 58 & $2.3 \mathrm{k}$ & 0.5 \\
$7 \times 7$ ASF & 108 & $4.3 \mathrm{k}$ & 0.9 \\
$3 \times 3$ Density operator & 36 & $2.2 \mathrm{k}$ & 0.4 \\
$5 \times 5$ Density operator & 95 & $6 \mathrm{k}$ & 1.2 \\
$7 \times 7$ density operator & 152 & $9.6 \mathrm{k}$ & 2.0 \\
\hline
\end{tabular}

Table $10 \mathrm{cpp}$ and frame processing rate of Associative Mesh (for $200 \times 200$ images $)$

\begin{tabular}{llll}
\hline Algorithm & $\mathrm{cpp}$ & $t(\mu \mathrm{s})$ & Frame rate \\
\hline Frame Difference & 0.5 & 1.024 & $977 \times 10^{3}$ \\
$\Sigma \Delta$ & 35 & 70 & $14.3 \times 10^{3}$ \\
ICM & 70 & 140 & $7.14 \times 10^{3}$ \\
Geodesic reconstruction & 0.46 & 0.94 & $1.07 \times 10^{6}$ \\
$3 \times 3$ Morphological opening & 0.19 & 0.39 & $2.58 \times 10^{6}$ \\
$7 \times 7$ Morphological opening & 0.44 & 0.91 & $1.11 \times 10^{6}$ \\
$3 \times 3$ Density operator & 0.32 & 0.65 & $1.55 \times 10^{6}$ \\
$7 \times 7$ Density operator & 10.21 & 20.9 & $47.8 \times 10^{3}$ \\
\hline
\end{tabular}

binary morphology) the computation time only depends on frequency of the retina. For the spatial processing (binary morphology), three different sizes are considered for the largest radius of the structuring element set used both by the alternated sequential filter and the density operator.

If we only consider the computation time, the overall time consumed by the PAR is approximately $3.5 \mathrm{~ms}$ per frame, among which $2 \mathrm{~ms}$ for the CNA and $1.5 \mathrm{~ms}$ for the algorithm itself. These times are measured for a control frequency of $5 \mathrm{MHz}$. This means that, if we discard the acquisition time (which can make sense for a PAR observing a strongly lighted scene, for which the $2 \mathrm{~ms}$ of the CNA are sufficient as acquisition time), then a frame rate of $285 \mathrm{images} / \mathrm{s}$ is attainable at $5 \mathrm{MHz}$. Conversely, if the frame rate of 25 images/s is sufficient, then the control frequency can be lowered to $440 \mathrm{kHz}$, thus reducing proportionally the computing power.

At $5 \mathrm{MHz}$, the computing power of the whole device (PAR + EPXA1 board) has been measured at less than 1 $\mathrm{W}$, from which only $100 \mathrm{~mW}$ is consumed by the PAR circuit and its cortex controller (external micro controller, Fig. 5) and the rest by the EPXA1 board. This means that the computing power of the PAR-based vision system can certainly be lowered significantly by developing specific controlling ASIC instead of using off-the-shelf development kit.

\subsection{Associative Mesh results}

The results provided in the following section were simulated using a $90 \mathrm{~nm}$ technology parameter. On the Associative Mesh, the ICM cpp is about 70 for one ICM relaxation (varying from 70 to 80 , depending on the degrees of SIMD and virtualization) and 35 for $\Sigma \Delta$.

For the morphological operator, the Associative Mesh cpp is higher than PowerPC G4 cpp because of 1-bit implementation of PLUS - association. But with SIMD distributed processing power, it has the higher frame processing rate, even with virtualization. 
The Mesh achieves spectacular performance. The bandwidth offered by its internal busses allow the Mesh to achieve a frame processing rate of 24,800 images/s. This number could, however, be impacted by the performances and/or synchronization with the video sensor. Another physical limitation is the number of incident photon impact(s) on the associated sensor.

\subsection{Synthesis benchmarks}

We only take into account the computation time and discard the acquisition time, the conversion time, the transfer time, and the power consumption of these operations. We are aware that results are a bit unfavorable to the PAR as the acquisition and conversion is integrated into itself contrary to the Mesh and the PowerPC. Right now, there is no way to get better results so the synthesis benchmarks will focus on the computation time to evaluate architecture performance. Considering the power consumption of a sensor-which is about $500 \mathrm{~mW}$ for both acquisition and conversion-the simulation and execution times will change but one order of magnitude between the PAR, the Mesh and the PowerPC performances will still exist.

Two configurations of benchmarks have been done (Table 11):

\#1 $\Sigma \Delta+$ Markovian relaxation (four iterations of ICM),

\#2 $\Sigma \Delta+$ morphological post-processing (geodesic reconstruction, $3 \times 3$ density or $3 \times 3 \mathrm{ASF}$, depending on the architecture).

In the configuration $\# 1, \Sigma \Delta$ is considered as a pre-processing algorithm used to provide a better initialization for ICM than classical Frame Difference algorithm. In configuration \#2, $\Sigma \Delta$ is a "stand alone" algorithm with a post processing step to remove the remaining noise. The choice of spatial regularization algorithm has been done to be coherent with the architecture capabilities, i.e.:

- geodesic reconstruction on the Associative Mesh, since it is the strongest algorithm, by far and its

Table 11 Benchmarks results for configurations \#1 and \#2

\begin{tabular}{lrll}
\hline Image size & PowerPC G4 & Retina & Mesh \\
\hline Configuration \#1: $\Sigma \Delta+4$ ICM & 1,178 & - & \\
Frame rate & 21 & - & 24,800 \\
Real-time Freq $(\mathrm{MHz})$ & 8,500 & - & 0.504 \\
Energy $(\mu \mathrm{J})$ & 3,436 & 667 & 201.6 \\
Configuration $\# 2: \Sigma \Delta+$ morpho & 7,300 & 188 & 68 \\
Frame rate & 2,900 & 150 & 27.1 \\
Real-time Freq $(\mathrm{kHz})$ & & & \\
Energy $(\mu \mathrm{J})$ & &
\end{tabular}

implementation is efficient on Associative Mesh (compared to the implementation of the other architectures)

- $3 \times 3$ ASF on the PAR, since ASF is the most efficient operator on the retina.

- $3 \times 3$ density on SWAR CPU, to get a complexity that is comparable to PAR complexity, keeping in mind that after optimizations, SWAR $5 \times 5$ and $7 \times 7$ operators are quite as fast as the $3 \times 3$ operator.

To assess the performance of the retina, we only take into account the processing time $(1.3+0.2=1.5 \mathrm{~ms})$ not the total time (acquisition + conversion + processing). The reason is that both acquisition and conversion times are unknown for the PowerPC G4 and the Associative Mesh. This leads to a frame rate of 667 images/s. The estimation of energy consumption is based on this assumption.

Table 12 presents the energy consumption of the three architectures for the configurations \#1 and \#2. We can notice that specialized architectures are by far more efficient than the general purpose processor-even with SWAR computation: performance ratios are all greater than $\times 10$. This also means that even a $50 \%$ error, about the estimation of PowerPC G4 power consumption, is definitively not a problem.

\subsection{Benchmark analysis}

Before concluding, we focus, for each architecture, on the impact of the optimizations and the efficiency of the implementation.

\section{RISC PowerPC G4}

- From a point of view of embedded system, Altivec is well-adapted to complex algorithm like ICM relaxation: the ratio with Associative Mesh is $\times 35.7$ for configuration \#1 and $\times 88.6$ for configuration \#2. That could lead people to redesign SIMD Mesh PE architecture with an Altivec-like SWAR Instruction Set Architecture. For example a sub-set with only integer and also with restriction within the cross-bar capabilities could be integrated on a FPGA.

- For RISC, SWAR is very efficient, since a complex and robust algorithm like those proposed in the

Table 12 Energy comparison for configurations \#1 and \#2

\begin{tabular}{lllll}
\hline Image size & 128 & 256 & 512 & 1,024 \\
\hline Configuration $\# 1:$ & $\Sigma \Delta+4$ ICM & & \\
G4/AM & $\times 36$ & $\times 42$ & $\times 139$ & $\times 155$ \\
Configuration & $\# 2:$ & $\Sigma \Delta+$ morpho & & \\
G4/PAR & $\times 14.4$ & $\times 18.0$ & $\times 61.3$ & $\times 72.1$ \\
G4/AM & $\times 89$ & $\times 107$ & $\times 329$ & $\times 395$ \\
PAR/AM & $\times 5.5$ & $\times 5.5$ & $\times 5.5$ & $\times 5.5$ \\
\hline
\end{tabular}


configuration 1 and 2, are running faster, after SIMDization, than naive Frame Difference.

- Another point for fair comparison, is the cache size of a RISC. We can see that the G4 is efficient (cpp low) for size up to $300 \times 300$. This means that for smaller size, the G4 efficiency is underestimated, from an embedded point of view, since it will work fine with smaller cache. Not only we can apply a down-clocking frequency for its embedded version, but we can also reduce its cache (both will decrease power consumption).

- Down-clocking for System on Chip: Altivec frequency could be as low as $10 \mathrm{MHz}$ for both configurations and for $128 \times 128$ and $256 \times 256$ images.

Retina

- The cost of the serial-bit $A L U$ is a problem for arithmetic operators. A 8-bit ALU would have a great impact on performance, but will also have a negative impact of size and power consumption of the retina. A material full adder may be a golden mean to have good arithmetic performance.

- Asynchronous logic and graph manipulation is a must have for specialized architecture, not only for low level operations, but also and especially for middle level operations with irregular processing like the morphological reconstruction. Next generation of artificial retina should integrate such kind of silicon graph management.

Associative Mesh

- Computation results show that the Associative Mesh is well suited for both configurations. Each sequence of algorithms takes advantage of one of the Mesh's architectural characteristics. For configuration 1, the massively parallel resources easily handle the amount of computation required by the ICM relaxation. For configuration 2 , the dynamic reconfiguration of the graph's structure allows to efficiently represent the objects, while the asynchronous implementation of global operations guarantees a fast processing of the geodesic reconstruction. In both cases, frame rates are quite spectacular.

- A remarkable aspect of the algorithms implementation on the Associative Mesh, in contrast with PowerPC G4 (and to a lesser extent, with retina) is that computation time is quasi-independent of the images' size or the detected object's shape.

- The major drawback is the hardware cost of the Mesh to process big images when compared to the other architectures. Still, vision SoC implementation of a $256 \times 256$ Associative Mesh is compliant with current technology and only requires 3 times more transistors than a PowerPC G4 for a $\times 20$ faster computation.

- With such performance, reducing the clock frequency by a factor 10 could still allow to process more than two thousand $256 \times 256$ images/s with a power consumption under $1 \mathrm{~W}$. The Associative Mesh could then be used in association with a HD camera on a SoC platform.

\section{Conclusion: future architectures}

We have presented the implementation of robust sets of operator for Motion Detection, based on Markov Random field, Sigma-Delta filtering and morphological operators like opening, density and Alternate Sequential Filter. These algorithms have been used to emphasize the intrinsic qualities and drawbacks of these architectures (Sect. 5.1) and then to envision the specification of future architectures, first with SWAR paradigm (Sect. 5.2), second with FPGA-based customization (Sect. 5.3) and finally with many-core reconfigurable processor (Sect. 5.4).

\subsection{Pros and cons of the three architectures}

- SWAR is efficient for low level algorithm. Currently used in RISC processor and also present and customized in some SoCs.

- Asynchronous Associative Networks. This model of computation is extremely efficient for both power consumption and intermediate levels algorithms. It is efficient for power consumption because asynchronism mechanism. It is also interesting for speed since, in our case, up to 40 asynchronous associative operators can be executed during 1 synchronous cycle. It is also efficient for intermediate level of processing because associative networks can be reconfigured and then, an operator can be applied through a graph, to any connected components. Any kind of irregular and CPU intensive algorithms can be handled efficiently, like geodesic reconstruction, watershed segmentation and of course, connected component labeling.

- Retinas are very low power embedded architecture. For tight integration and an optimised connection between sensor and calculator, retinas outperform SoC and Vision SoC systems like FPGA + sensor. But, right now they are limited to regular processing. Integrating an associative network inside a retina will allow to use such a kind of machine for intermediate level algorithm. So a quite complete image processing chain could fit into a high parallel and versatile system. 


\subsection{SWAR enhancement}

Nowadays, the two main solutions to computer architecture limitations are: increasing RISC performance or customizing FPGA.

When RISCs have replaced CISCs using architectural optimizations like pipeline, registers and cache, the RISC motto was more instructions per cycle, because they were using less complex instructions that can be fetched, decoded and executed faster than CISCs can. As at this time, it was commonly accepted that clock frequency can go higher and higher. The easiest way, thanks to the technology, was to increase the clock frequency. At the same time two evolutions of RISC were released: the superscalar architecture (multi ALU/FPU per chip) and the VLIW (Very Long Instruction Word) like the Intel Itanium or the Texas Instrument C6x DSP family. But since a few years, clocks frequency does not increase as much as before. The new RISC motto could be "more instruction per second". The General Purpose solution is the multicore approach (see Sect. 5.4) And the Domain Specific solution is SWAR extension. As we can see in Table 7, the speedup provided by Altivec — up to $\times 60$ —released in 1998 is by far, greater than the current number of cores inside a processor in 2008. As SWAR implementation requires few transistors because of the very simple control structure due to SIMD model, one very efficient way to increase performance could be "more SWAR into RISC"

- longer registers: 256 bits or even 512 bits, to process more data per cycle,

- more smart instructions: Altivec has a very useful vector permutation unit that provides powerful instructions like vec_sel that is an aesthetic way to perform a SIMD if then else condition (replacing masks computations and combinations) or vec_perm that can permute data with any kind of pattern (SSE can only do regular patterns of interlacing). vec_perm is used for computing unaligned vectors, matrix multiplications and even for sorting data. Such a kind of unit should be present in any SWAR architecture,

- more specialized or dedicated instructions like Altivec vec_sum, vec_msum that performs reduction into a register and the SSE2_mm_sad_epu8(a,b) that performs a sum of absolute difference (SAD) between two registers. This instruction is used in every correlation algorithm based on block-matching. Adding such an instruction has been studied into [29].

\subsection{FPGA-based customization}

Processor customization, as defined for reconfigurable architectures [39] and embedded systems, have to be explored. A customizable processor is a General Purpose Processor (GPP) embedded into a FPGA which cores can be enhanced. Most major FPGA manufacturers now provide solutions with softcore customizable FPGA (NIOS 2 for Altera, microblaze for Xilinx). Such technologies have room for improvements like adding new instructions, new customized format [37] for specific domain application [38] but also new dedicated blocks. With a compiler like $\mathrm{C} 2 \mathrm{H}$ for Altera FPGA or DIME-C for Xilinx, a complete C function can be compiled into a VHDL block and be directly called inside a $\mathrm{C}$ code. GPP and its accelerators can then be seen as a full system on a chip. With these two levels of customization (instruction and hardware function) one can envision to add a new specialized instruction at the $\mathrm{C}$ level or new hardware function. A new instruction could be $\mathrm{b}=\operatorname{sigmaDelta}(\mathrm{a}, \mathrm{b})$ that compares $\mathrm{a}$ and $\mathrm{b}$ and increment/decrement $\mathrm{b}$ according to the result of the comparison. Such a function will remove if then else structure that stalls/flushes the pipeline. On the other hand an hardware function could implement a morphological operator. Such hardware implementation can be much more faster than the sequential execution of the instructions that compose it, as no more "register to register" stage is required at each cycle like it is the case for pipeline execution. One of the best example of processor customization (not softcore but ASIP) is the Tensilica Xtensa architecture [37].

\subsection{Many-core reconfigurable processor}

If classic systems are able to race against Moore's Law (bigger caches, more complex branch predictors, more hardware optimizations), they are slowly but steadily losing the efficiency race. The amount of transistors involved in those systems keep increasing, but most of them are only used to stay on par with Moore's Prediction. They could be used more efficiently if GPP were no so "General Purpose" but also target some specific domains like computer vision or multimedia. The solution to this problem is brought by recent technology advances by combining both above solutions: designing reconfigurable parallel processor.

This leads to the fact that different models of computation have to be implemented in order to fit a given domain constraints. For example, the PAR can execute various regular algorithms in a very fast and efficient way even if the PAR itself is only composed of simple processors with few bits of memory. Similarly, the Mesh, thanks to its asynchronous network can handle irregular algorithms. Another example of such a processor is the PIMM [25]. PIMM is dedicated to morphological operations and use, an explicit hardware queue model to execute algorithms-like geodesic reconstruction or watershed 
segmentation-faster than a GPP, which are the most used architecture for such tasks but are, in fact, less efficient.

Currently, multi-cores approach is the leading solution for Thread Level Parallelism. But these processors are designed for regular processing, irregular processing still being out of their range. This also applies to CELL processor and GPU: Cell is just a 9-core heterogeneous RISC processor and GPU-from Nvidia or ATI-are still dedicated to regular processing even if they are going to be more flexible when used with a Stream Computing language-CUDA [11] and Brook GPU [6].

Next generation will include specialized/dedicated logic to tackle the problem of GPP inefficiency. Adding a custom part of logic (100-200 millions of transistors) is definitively no more a problem, compared to the total size of a CPU (800 millions of transistors for current Intel quad core). One of the most promising architecture of this kind is the Intel Polaris/Larrabee architecture from Terascale project [41]. Polaris has a hierarchical bus to connect PEs together, PEs include an 512-bit SWAR unit and can be reconfigured, for $3 \mathrm{D}$ graphic processing or cryptography.

We believe that adding a custom part of logic into GPP (100-200 millions of transistors is now just a part of) dedicated to irregular processing would be a solution to the problem of GPP inefficiency.

Acknowledgment We wish to thanks Joel Falcou for his valuable help.

Open Access This article is distributed under the terms of the Creative Commons Attribution Noncommercial License which permits any noncommercial use, distribution, and reproduction in any medium, provided the original author(s) and source are credited.

\section{References}

1. Azencott, R.: Simulated Annealing: Parallelization Techniques. Wiley, New York (1992)

2. Bellon, A., Derutin, J.-P., Heitz, F., Ricquebourg, Y.: Real-time collision avoidance at road-crossings on board the PrometheusProLab 2 vehicle. Intell Vehicles, pp. 56-61 (1994)

3. Biancardi, A., Segrovia-Martinez, M.: Adaptative segmentation of MR axial brain images using connected components. In: International Workshop on Visual Form, Capri, pp. 295-302 (2001)

4. Bouthémy, P., Lalande, P.: Recovery of moving object in an image sequence using local spatiotemporal contextual information. Opt Eng 32(6), 1205-1212 (1993)

5. Blelloch, G.: Vector Models for Data-Parallel Computing. MIT Press, Cambridge (1990)

6. BrookGPU: http://ati.amd.com/technology/streamcomputing

7. Caplier, A., Bonnaud, L., Chassery, J.-M.: Robust fast extraction of video objects combining frame differences and adaptive reference image. Int Conf Image Process, pp. 785-788 (2001)

8. Ceccarelli, M., Petrosino, A.: A parallel fuzzy scale-space approach to the unsupervised texture separation. Pattern Recognit Lett 23(5), 557-567 (2002)
9. Cohen, F.S., Cooper, D.B.: Simple parallel hierarchical and relaxation algorithms for segmenting non-causal Markovian random fields. IEEE PAMI 9(2), 195-219 (1987)

10. Cray X1: http://www.cray.com/products/x1/specifications.html

11. CUDA: http://www.nvidia.com/cuda

12. Denoulet, J., Mérigot, A.: System on chip evolution of a SIMD architecture for image processing. In: Computer Architecture and Machine Perception, New Orleans, 12-16 May 2003. doi: 10.1109/CAMP.2003.1598175

13. Denoulet, J., Mérigot, A.: Evaluation of a SIMD architecture dedicated to image processing. Global Signal Process (2004)

14. Denoulet, J., Mostafaoui, G., Lacassagne, L., Mérigot, A.: Implementing motion Markov detection on General Purpose Processor and Associative Mesh. In: Computer Architecture and Machine Perception, Palermo, pp 288-293 (2005)

15. Diefendorff, K., Dubeyn, P.K., Hochsprung, R., Scales, H.: Altivec extension to PowerPC accelerates media processing. In: IEEE Micro (2000)

16. Ducourthial, B., Merigot, A.: Parallel asynchronous computations for image analysis. Proc IEEE 90(7), 1218-1229 (2002)

17. Ducourthial, B., Constantinescu, G., Merigot, A.: Implementing image analysis with a graph-based parallel computing model. Computing. In: Supplementum, GbR'97, Workshop on Graph based Representation, Lyon, pp. 111-121 (1997)

18. Dudek, P.: An asynchronous cellular logic network for triggerwave image processing on fine-grain massively parralel arrays. IEEE Trans Circuits Syst II Express Briefs 53(5), 354-358 (2006)

19. Dulac, D., Merigot, A., Mohammadi, S.: Associative meshes: a new parallel architecture for image analysis applications. In: Computer Architecture and Machine Perception, News Orleans, pp 393-399, 15-17 December 1993

20. Forchheimer, R., Astrøm, A.: Near-sensor image processing: a new paradigm. IEEE Trans Image Process 3, 736-746 (1994)

21. Daniel Hillis, W., Tucker, L.W.: The CM-5 connection machine: a scalable supercomputer. Commun ACM 36(11), 31-40 (1993)

22. Galilee, B., Mamalet, F., Renaudin, M., Coulon, P.Y.: Parallel asynchronous watershed algorithm-architecture. IEEE Trans Parallel Distrib Syst 18(1), 44-56 (2007)

23. Gies V., Bernard T.: Increasing interconnection network connectivity for reducing operator complexity in asynchronous vision systems. In: International Conference on Discrete Geometry for Computer Imagery, Poitiers, pp.1-10 (2005)

24. Gies, V., Bernard, T.M.: Tree extension of micro-pipelines for mixed synchronous-asynchronous implementation of regional image computations. In: Proceedings of SPIE, vol 5677, Sensors and Camera Systems for Scientific and Industrial Applications VI, SPIE (2005)

25. Klein, J.-C., Lemonnier, F., Gauthier, M., Peyrard, R.: Hardware implementation of the watershed zone algorithm based on a hierarchical queue structure. In: IEEE Workshop on Nonlinear Signal and Image Processing, Neos Marmaras (1995)

26. Komuro, T., Ishii, I., Ishikawa, M., Yoshida, A.: A digital vision chip specialized for high-speed target tracking. IEEE Trans Electron Devices 50(1), 191-199 (2003)

27. Lacassagne, L., Milgram, M., Garda, P.: Motion detection, labeling, data association and tracking, in real-time on RISC computer. In: International Conference on Image Analysis and Processing, Venise, pp 520-525 (1999)

28. Lalande, P., Bouthemy, P.: A statistical approach to the detection and tracking of moving objects in an image sequence. Eur Signal Process Conf 2, 947-950 (1990)

29. Limousin, C., Sebot, J., Vartanian, A., Drach, N.: Architecture optimization for multimedia application exploiting data and thread-level parallelism. J Syst Arch EUROMICRO J 51(1), 1527 (2005) 
30. Lohier, F., Lacassagne, L., Garda, P.: A New Methodology to Optimize DMA Data Caching: Application toward the Real-time Execution of an MRF-based Motion Detection Algorithm on a multi-processor DSP. International Conference on Signal Processing Applications and Technology, March 1999, Phoenix USA

31. Manzanera, A., Richefeu, J.: A robust and computationally efficient motion detection algorithm based on Sigma-Delta background estimation. In: Proceedings Indian Conference on Computer Vision, Graphics and Image Processing, Kolkata (2004)

32. Manzanera, A., Richefeu, J.: A new motion detection algorithm based on Sigma-Delta background estimation. Pattern Recognit Lett 28(3), 320-328 (2007)

33. Mérigot, A.: Associative nets model: a graph based parallel computing model. IEEE Trans Comput 46(5), 558-571 (1997)

34. Mérigot, A., Zavidovique, B.: Image analysis on massively parallel computers: an architectural point of view. Int $\mathrm{J}$ Pattern Recognit Image Anal 6(3), 387-399 (2002)

35. Nadrag, P., Manzanera, A., Burrus, N.: Smart retina as a contourbased visual interface. In: ACM Distributed Smart Cameras Workshop, DSC'06, Boulder (2006)

36. Sébot, J., Drach-Temam, N.: Memory bandwidth: the true bottleneck of SIMD multimedia performance on a superscalar processor. In: Proceedings of the 5th International Euro-Par Conference on Parallel Processing, pp 439-447, Springer, Berlin (2001)

37. Piskorski, S., Lacassagne, L., Bouaziz, S., Etiemble, D.: Customizing CPU instructions for embedded vision systems. IEEE Comput Arch Mach Percept Sensors (2006)

38. Piskorski, S. , Lacassagne, L., Kieffer, M., Etiemble, D.: Efficient floating point interval processing for embedded systems and applications. Int Symp Sci Comput Comput Arithmetic Valid Numer (2006)

39. List of reconfigurable architectures http://www.site.uottawa. $\mathrm{ca} / \sim \mathrm{rabielmo} /$ personal/rc.html

40. Rodriguez-Vazquez, A., Linan-Cembrano, G., Carranza, L., Roca-Moreno, E., Carmona-Galan, R., Jimenez-Garrido, F., Dominguez-Castro, R., Espejo Meana, S.: ACE16k: the third generation of mixed-signal SIMD-CNN ACE chips toward VSoCs. IEEE Trans Circuits Syst 51(5), 851-863 (2004)

41. Intel Tera-scale project: http://techresearch.intel.com/articles/ Tera-Scale/1449.htm

42. Zavidovique, B., Stamon, G.: Bilevel processing of multilevel images. In: Proceedings PRIP'81, Dallas (1981)

\section{Author Biographies}

Lionel Lacassagne is an assistant professor in Fundamental Electronics Institute (IEF), University of Paris Sud (France). His research areas deal with High Performance Computing and Image Processing applied to embedded systems and especially the benchmarking of vision systems. The research activities are done in AXIS team (Architecture, Control, Communication, Vision, Systems).

Antoine Manzanera is an assistant professor in the Electronics and Computer Science Lab. at ENSTA, Paris (France). His activity field is Image Processing and Computer Vision algorithmics, particularly for Real-time and Embedded systems. His research interests are discrete geometry, mathematical morphology, motion analysis, and image models.

Julien Denoulet is an assistant professor at University Pierre and Marie Curie in Paris (France). He received his $\mathrm{PhD}$ in 2004 from University of Paris-Sud, working on massively parallel architectures for image processing. His current activities take place in the SYEL (Electronic Systems) group and deal with SoC-AMS modeling and methodologies for design exploration on reconfigurable SOC platforms.

Alain Merigot is professor of Computer Engeneering at the University of Paris Sud in Orsay (France) and researcher at the Fundamental Electronics Institute and Digiteo Labs. His research interests are computer architecture and image processing. 